\title{
Tite: Conserved cysteines in titin sustain the mechanical function of cardiomyocytes
}

One sentence summary: Mutations targeting cysteines key to the mechanoredox control of titin cause human dilated cardiomyopathy

Authors: Elías Herrero-Galán ${ }^{1, \pi}$, Fernando Domínguez ${ }^{2,3 \pi}$, Inés Martínez-Martín ${ }^{1}$, Cristina SánchezGonzález $^{1}$, Natalia Vicente ${ }^{1}$, Laura Lalaguna ${ }^{1}$, Elena Bonzón-Kulichenko ${ }^{1,3}$, Enrique Calvo ${ }^{1,3}$, Esther González-López ${ }^{2,3}$, Marta Cobo-Marcos ${ }^{2,3}$, Belén Bornstein ${ }^{2}$, Ana Briceño ${ }^{2}$, Juan Pablo Ochoa ${ }^{2,4}$, Jose Maria Garcia-Aznar ${ }^{4}$, Carmen Suay-Corredera ${ }^{1}$, Maria Rosaria Pricolo ${ }^{1}$, Ángel Fernández-Trasancos ${ }^{1}$, Diana Velázquez-Carreras ${ }^{1}$, Claudio Badía Careaga ${ }^{1}$, Belén Prados ${ }^{1,3}$, Francisco Gutiérrez-Agüera ${ }^{1}$, Mahmoud Abdellatif ${ }^{5}$, Simon Sedej, ${ }^{5,6,7}$, Peter P. Rainer ${ }^{5,7}$, David Giganti ${ }^{8}$, Giovanna Giovinazzo ${ }^{1}$, Juan A. Bernal ${ }^{1}$, Raúl Pérez-Jiménez ${ }^{9,10}$, Torsten Bloch Rasmussen ${ }^{11}$, Thomas Morris Hey ${ }^{12}$, Inmaculada Vivo-Ortega $^{13}$, Jesús Piqueras-Flores ${ }^{13}$, Enrique Lara-Pezzi ${ }^{1,3}$, Jesús Vázquez ${ }^{1,3}$, Pablo GarciaPavia $^{2,3,14, *}$, Jorge Alegre-Cebollada ${ }^{1, *}$

Affiliations:

${ }^{1}$ Centro Nacional de Investigaciones Cardiovasculares (CNIC), Madrid, Spain

${ }^{2}$ Heart Failure and Inherited Cardiac Diseases Unit, Department of Cardiology, Hospital

Universitario Puerta de Hierro Majadahonda, Madrid, Spain

${ }^{3}$ CIBER de Enfermedades Cardiovasculares (CIBERCV), Madrid, Spain

${ }^{4}$ Health in Code, A Coruña, Spain

${ }^{5}$ Division of Cardiology, Medical University of Graz, Graz, Austria

${ }^{6}$ Faculty of Medicine, University of Maribor, Maribor, Slovenia

${ }^{7}$ BioTechMed Graz, Graz, Austria

${ }^{8}$ Department of Biochemistry \& Molecular Pharmacology and Institute for Systems Genetics, NYU

Langone Health, New York, New York, United States

${ }^{9}$ CIC nanoGUNE BRTA, San Sebastian, Spain

${ }^{10}$ Ikerbasque Foundation for Science, Bibao, Spain

${ }^{11}$ Department of Cardiology, Aarhus University Hospital, Aarhus, Denmark

${ }^{12}$ Department of Cardiology, Odense University Hospital, University of Southern Denmark, Odense, Denmark

${ }^{13}$ Department of Cardiology, Hospital Universitario de Ciudad Real, Ciudad Real, Spain

${ }^{14}$ Universidad Francisco de Vitoria (UFV), Pozuelo de Alarcón, Spain

${ }^{\text {II }} \mathrm{EHG}$ and FD contributed equally and are joint first authors.

* PGP and JAC are co-corresponding authors: pablogpavia@yahoo.es (Twitter: @dr_pavia); jalegre@cnic.es (Twitter: @ AlegreCebollada) 


\section{Abstract:}

2 The protein titin determines cardiomyocyte contraction and truncating variants in the titin gene (TTN)

3 are the most common cause of dilated cardiomyopathy (DCM). Different to truncations, missense

4 variants in $T T N$ are currently classified as variants of uncertain significance due to their high frequency

5 in the population and the absence of functional annotation. Here, we report the regulatory role of

6 conserved, mechanically active titin cysteines, which, contrary to current views, we uncover to be

7 reversibly oxidized in basal conditions leading to isoform- and force-dependent modulation of titin

8 stiffness and dynamics. Building on our functional studies, we demonstrate that missense mutations

9 targeting a conserved titin cysteine alter myocyte contractile function and cause DCM in humans. Our

10 findings have a direct impact on genetic counselling in clinical practice. 
Main text:

\section{INTRODUCTION}

3 Titin is a fundamental protein for the contractile function of striated myocytes since it provides

4 structural support and sets the stiffness of sarcomeres (1-3). Not surprisingly, the mechanical properties

5 of titin are exquisitely modulated both transcriptionally through specific alternative mRNA splicing (4,

6 5), and posttranslationally via biochemical modifications such as phosphorylation (6). Truncating

7 variants in the titin gene (TTN) are the main cause of dilated cardiomyopathy (DCM), a disease that is

8 the most frequent trigger of heart failure in the young and of heart transplantation worldwide (7-11).

9 However, the primordial pathomechanisms remain elusive, reflecting our incomplete knowledge of the

10 function of titin (12-14). Akin to other sarcomeric proteins in which both truncations and missense

11 variants lead to cardiomyopathy (9), it has been speculated that rare missense mutations in TTN could

12 be the cause of DCM in some of the $\sim 50 \%$ genotype-negative patients $(7,15,16)$. Indeed, numerous

13 titin missense variants can be identified in DCM individuals, although at a frequency that is not

14 significantly higher than in the general population (17). Hence, when rare missense variants are identified in $T T N$, they are currently classified as variants of unknown significance. It is conceivable though that population genetics analyses have limited power to set apart true pathogenic mutations

17 from a background of phenotypically silent $T T N$ missense variants. Here, we propose an alternative approach by which we first identify conserved residues that are important for the function of titin, which we then screen for pathogenic missense variants in DCM patients. We have focused on a group of structurally conserved cysteine residues whose modification has been suggested, on the basis of in

21 vitro evidence, to modulate the mechanical properties of titin (18). Following our strategy, we show 22 the in vivo relevance of cysteine-based regulation of titin mechanics and unequivocally demonstrate 23 that rare TTN missense variants cause DCM in humans.

\section{RESULTS}

26 Titin cysteines are reversibly oxidized in basal conditions 
1 The mechanical properties of titin stem from force-dependent conformational changes of polypeptide

2 regions that belong to the extensible I-band region of the protein (Figure 1A). These conformational

3 changes include extension and entropic recoil of the serially linked immunoglobulin-like (Ig) domains

4 and the random-coil N2Bus and PEVK regions, and Ig domain unfolding and refolding transitions (19).

5 Interestingly, the I-band of titin is rich in cysteine residues (18, 20), many of which appear at

6 structurally and evolutionary conserved positions within Ig domains (Figure 1B-F, Supplementary

$7 \quad$ Figure S1, Supplementary File S1). In vitro experiments have shown that oxidation of these cysteines

8 has major mechanical consequences. For instance, disulfide bonds established by the triad of conserved

9 cysteines B, F and G in Ig domains (98\% mean evolutionary conservation, Figure 1B,C,E) stiffen titin

10 via reduction of the protein contour length (21), while S-thiolation of cysteines 47 and 63 (94\% mean

11 evolutionary conservation, Figure 1B,D,E) lead to titin softening through Ig folding inhibition (22).

12 These five structurally conserved cysteines are more evolutionary conserved than cysteines appearing

13 in other positions in Ig domains ( $97 \%$ vs $89 \%$ mean evolutionary conservation, Figure 1F). The N2Bus

14 region also contains cysteines that can stiffen titin through disulfide bond formation (23), albeit they

15 are less evolutionary conserved than Ig domain cysteines (53\% mean conservation, Figure 1F,

16 Supplementary Figure S1C). Beyond the remarkable conservation of I-band titin's cysteine residues

17 and their role in the evolution of the protein in vertebrates (24), the in vivo relevance of redox mechanical modulation of titin is supported by limited data on the global oxidation of the protein (25$28)$, the effects of redox-active molecules on striated muscle mechanics $(22,23,29,30)$, and the disulfide-compatible location of the majority of structurally conserved cysteines of titin $(21,31)$.

21 However, the extent and location of native titin oxidations remain unexplored, limiting our understanding of the impact of oxidative modifications on the mechanical function of the protein.

24 To measure the oxidation state of native titin, we first developed an SDS-PAGE-based assay that 25 exploits thiol chemistry reactions to block reduced cysteines with an alkylating agent and label 26 reversibly oxidized cysteines with the fluorophore monobromobimane (mBBr) (Figure 2A) (32). The 
1 assay takes advantage of unequivocal identification of titin as the slowest-migrating protein in low-

2 percentage-acrylamide SDS-PAGE gels $(33,34)$. We verified that the $\mathrm{mBBr}$ fluorescence signal of titin

3 cysteines is linearly dependent on the amount of lysate analyzed (Figure 2B, Supplementary Figure

4 S2) and used Coomassie staining (Figure 2C, Supplementary Figure S2) to get normalized oxidation

5 measurements that are independent of the amount of protein loaded on the gel (Figure 2D). The initial

6 alkylating step is done in denaturing conditions to block all reduced cysteines. To limit artifactual

7 oxidation signal, this step needs to be fast and complete. We chose N-ethylmaleimide (NEM) as the

8 alkylating agent because of its superior reaction kinetics and its high solubility in aqueous buffers (27,

9 35). Using murine cardiac samples, we found that the oxidation signal of titin plateaus when the

10 concentration of NEM in the lysis buffer is above $5 \mathrm{mM}$ (Figure 2E), suggesting that these conditions

11 result in minimal artifactual oxidation. To further ensure efficient thiol blockage, in all our subsequent

12 experiments we perfused myocardial tissue with PBS containing $50 \mathrm{mM}$ NEM immediately after

13 sacrifice. The same high NEM concentration was kept during lysis. As an additional preventive

14 measure, we subtracted the $\mathrm{mBBr}$ signal of samples not incubated with DTT to account for potential

15 reduced thiols refractory to NEM blockage (see Methods). Using our in-gel mBBr fluorescence assay,

16 we observed that the extent of reversible cysteine oxidation of titin is $\sim 4$ times higher than that of

17 myosin, a partner protein of titin in the sarcomere (Figure $\mathbf{2 F}$ ), confirming that a fraction of titin

18 cysteines are constitutively oxidized in cardiac tissue.

\section{Titin oxidation is boosted upon birth}

21 Having demonstrated that titin is oxidized in vivo, we set out to test if titin oxidation is modulated

22 physiologically. With this aim, we turned to the perinatal model. In mammals, birth is accompanied by

23 exposure to high $\mathrm{O}_{2}$ concentration, which impacts on the function of many cells and organs including

24 the heart (36). Indeed, during early postnatal development, cardiomyocytes arrest cell cycle following

25 activation of oxidative signals (37). We hypothesized that increased oxidative conditions could result

26 in higher levels of titin oxidation upon birth, which we set out to test using the in-gel $\mathrm{mBBr}$ fluorescence 
1 assay. In order to increase statistical power, in the experiments we included control proteins that

2 enabled averaging data from multiple samples (Supplementary Figure S3A, see Methods). Since the

3 proportion of titin isoforms in the heart changes during postnatal development $(4,38)$, and these

4 isoforms have different proportion of cysteines (21), we normalized the results according to the cysteine

5 density at each developmental stage (Figure 3, Supplementary Figure S3B, see Methods). Supporting

6 our initial hypothesis, we found that cardiac titin oxidation is $43 \pm 15 \%$ (mean \pm SEM) higher in newborn

7 (P0) mice than in E18.5 embryos, while we detected no significant difference between P0 and adult

8 samples.

9

10 Preferential oxidation of I-band cysteines

11 To characterize the landscape of reversible titin cysteine oxidations, we resourced to mass spectrometry

12 (MS). We analyzed P0 samples because they contain a high proportion of the cysteine-rich N2BA titin

13 isoform. Fluorescent titin bands were sliced from SDS-PAGE gels and treated with trypsin. The

14 resulting peptides were subjected to LC-MS analysis and MS/MS spectra were searched against a

15 mouse proteome database. As expected from the full titin band separation in 3.5\% SDS-PAGE gels, 79

$16 \pm 1 \%$ (3 replicates) of the identified species corresponded to titin-derived peptides, even when using a

17 relaxed identification criterion (5\% False Discovery Rate, FDR) (Figure 4A). Titin coverage in these

18 searches was $33 \pm 3 \%$ (Figure 4B). To maximize the coverage of cysteine peptides, we followed an

19 alternative, more targeted strategy (39). Since most detected peptides in the search against the full

20 proteome originate from titin, we repeated the search against a database containing only titin and all

21 identified cysteine peptides were validated using Vseq (40). Similar to the in-gel fluorescence method,

22 we excluded $\mathrm{mBBr}$-derivatized peptides when also detected in -DTT control samples, resulting in a

23 final $45 \%$ aggregated cysteine coverage at a FDR $<2 \%$ (see Methods).

24

25 In the MS experiments, we detect reduced and oxidized cysteines from the mass shifts associated to

$26 \mathrm{NEM}$ and $\mathrm{mBBr}$ modifications, respectively (Figure $\mathbf{2 A}$ ). To obtain a global picture of oxidation, we 
1 have calculated an oxidation score (OS) for each cysteine position in the alignment of the Ig domains

2 of titin (Figure 4C, see Methods). OS data show that titin cysteines of the mechanically active I-band

3 are significantly more oxidized than those belonging to the A-band (Figure 4D). In addition, cysteines

4 of the disulfide-competent triad BFG (in particular cysteines F and G) are detected as oxidized more

5 frequently than other cysteines in titin (Figure 4C,E,F). Unfortunately, we did not detect cysteine-

6 containing peptides from the N2Bus region of murine titin, so its native oxidation state could not be

7 determined.

9 Titin oxidation in human hearts

10 We next examined the landscape of cysteine oxidation in human cardiac titin using left ventricular 11 snap-frozen samples from two non-failing donor hearts. These samples were processed and analyzed

12 by LC-MS following the same approach used for mouse samples. $74 \pm 3 \%$ of identified peptides were

13 derived from titin when the MS/MS search was done against the human proteome database at a $5 \%$

14 FDR (Figure 5A). In these searches, titin coverage was $37 \pm 6 \%$ (Figure 5B). As in experiments using mouse samples, we run targeted searches against a database containing only human titin and the resulting cysteine peptides were validated with Vseq. $\mathrm{mBBr}$-derivatized peptides also detected in -

17 DTT samples were excluded from analysis, leading to a final $39 \%$ aggregated cysteine coverage at a

18 FDR $<2 \%$. Results show similar levels of global oxidation in humans and in mice (global OS are 0.1

19 and 0.4, respectively, Figure 5C). As in mice, cysteines of the I-band are significantly more oxidized

20 than those of the A-band (Figure 5D) and the OS of structurally conserved cysteines in Ig domains

21 tends to be higher than that of non-structurally conserved cysteines (Figure 5C,E). Similar to results

22 with murine samples, cysteine F shows the highest OS among disulfide-competent positions, although

23 in human samples the I-band-specific cysteine 47 has the highest OS among the structurally conserved

24 cysteines (Figure 5C,F). In one of the human samples, we detected two cysteines belonging to the

25 N2Bus region (Cys4083 was detected as reduced, and Cys4124 was found in oxidized and reduced 
states). In summary, MS results indicate that reversible oxidations are also present in native titin from non-failing human myocardium.

\section{Modulation of titin mechanics by redox modifications}

Our results show that evolutionary and structurally conserved cysteines of the I-band of titin are oxidized in vivo. We find reversible oxidations both in disulfide-competent cysteines and in unpaired cysteines that can establish S-thiolation adducts. These modifications have been proposed to induce opposite mechanical effects $(18,21,22)$, an observation that may contribute to explain the different modulation of titin-based striated muscle stiffness under specific redox challenges $(22,23,29,30)$. To illustrate the range of regulation of titin mechanics by cysteine oxidation, we built on previous Monte Carlo simulations (22) to integrate all known mechanical effects of redox posttranslational modifications in titin Ig domains. These include reduction of contour length by disulfide bonds (21), higher unfolding and folding rates of disulfide-containing domains $(21,41)$, and higher unfolding rates and hampered folding of S-thiolated domains (22). In our simulations, we tuned unfolding and refolding rate constants to qualitatively reproduce the recently described unfolding/folding dynamics of native titin (33) (Supplementary Note S1, Supplementary Figure S4A). In the simulations, a virtual human I-band titin is subject to $1 \mathrm{~Hz}$ triangular force pulses between 0 and a predefined peak force, and the resulting length of titin is measured (Figure 6A,B). During the extension/relaxation cycles, titin domains unfold and refold stochastically according to their folding and unfolding rates, which are dependent on their redox state. At $t=0 \mathrm{~s}$, all domains are folded and, as the simulations proceed, a fraction of domains transition to the unfolded state resulting in longer titin lengths at peak force (Figure 6C,D). The simulation time was long enough to reach steady-state lengths at all peak forces (Figure 6C,D, Supplementary Figure S4B-E). 
$1 \mathrm{pN}$, the effect of disulfides reverses leading to overall titin stiffening (Figure 6D). At this high peak

2 force, S-thiolation maintains its softening effect. Additive mechanical modulation occurs if both

3 oxidative modifications are present in titin simultaneously (Figure 6C,D). To have a broader view of

4 the extent of titin softening/stiffening induced by redox modifications, we did simulations at a range of

5 peak forces and calculated the ratio of titin steady-state peak lengths between the oxidized and the

6 reduced conditions. Results show that the softening effect of S-thiolation remains fairly constant,

7 whereas at 50-80 pN peak force, the contribution of disulfides transitions from softening to stiffening

8 (Figure 6E, Supplementary Figure S4F). This dual behavior stems from the fact that disulfides favor

9 mechanical unfolding of Ig domains (softening effect), while also reducing the contour length of

10 unfolded domains and increasing folding rates (stiffening effects). At low peak forces in which Ig

11 domain unfolding rates are low, the softening effect is more prominent; while at high peak forces in

12 which Ig unfolding is more frequent, the stiffening effects prevail. Beyond modulation of steady-state

13 titin stiffness, our simulations also illustrate that both disulfides and S-thiolations induce a more

14 dynamic state of titin by favoring Ig domain unfolding reactions, particularly at low forces (Figure 6F,

15 Supplementary Figure S4G).

17 In the human heart, the short N2B isoform is expressed to similar levels as the longer and softer N2BA

18 (42, 43). Since alternative splicing occurs at the region of titin with the highest density of cysteines

(Figure 1B), we also ran Monte Carlo simulations for the N2B isoform of titin. In contrast to the results obtained with N2BA, we find that disulfides do not induce softening of N2B titin at any peak force

21 (Figure 6G, Supplementary Figure S4H-N). Interestingly, S-thiolation softens N2B titin to a greater extent (20\% vs. $10 \%$ for N2BA titin at $50 \mathrm{pN}$ peak force, Figure 6E,G), reflecting the higher density of S-thiolation-competent Ig domains in N2B (Supplementary Table S1). For the same reason, the extent of modulation of titin dynamics by redox modifications is also different in N2B and N2BA titins 
2 Our MS results were scarce with regards to the oxidation state of the cysteines in the N2Bus region if 3 titin. Monte Carlo simulations show that potential disulfides in the N2Bus (23) would boost the overall 4 stiffening effect of this redox modification, especially in the short N2B isoform (Supplementary

5 Figure S4P,Q). Indeed, under conditions in which N2Bus cysteines form disulfides, disulfides always 6 stiffen titin (Supplementary Figure S4P,Q). Taking together all the results from the Monte Carlo 7 simulations, we conclude that reversible redox modifications have profound effects in the mechanics 8 and dynamics of titin, in a manner that is highly dependent on the specific modification, the isoform of $9 \quad$ titin and the applied force protocol.

11 Missense variants that affect conserved Cys3575 cause DCM

12 Having shown the functional relevance of titin conserved cysteines, we screened patients followed at 13 the Heart Failure and Inherited Cardiac diseases Unit of Hospital Universitario Puerta de Hierro 14 Majadahonda (Madrid, Spain) for variants targeting these residues. We identified a clear match in a patient who carried a Chr2(GRCh38):g.178741559A>T; p.Cys3575Ser variant (Figure 7A) (protein numbering according to Uniprot Q8WZ42). Cys3575, which is $100 \%$ conserved in 27 species ranging

17 from zebrafish to human (Supplementary Figure S5A), is the conserved cysteine F of the cardiac specific I21 domain (Figure 7B) and shows OS =1 both in human (Figure 5C) and mice (the equivalent position in mouse titin is Cys3581).

21 The proband was a male who underwent cardiac transplantation at 57 years of age and who carried no 22 other pathogenic or likely pathogenic genetic variants in DCM-causing genes. 12/14 (86\%) of the 23 p.Cys3575Ser carriers in the family had DCM phenotype with a median age at DCM diagnosis of 33 24 years (interquartile range, IQR: 18-45) (Figure 7A). Mean left ventricular ejection fraction (LVEF) 25 was $43 \pm 6 \%$ and mean left ventricular end-diastolic diameter (LVEDD) was $57 \pm 4 \mathrm{~mm}$, as determined 26 by echocardiography (errors are SD). The two individuals who had the missense variant and did not 
1 show DCM phenotype were a 38 year-old man and a 64 year-old woman, although LVEF was in the

2 lower limit of normal range in both cases (50-55\%). A total of 22 additional relatives were non-carriers

3 of the variant and all showed a normal phenotype (median age 51 years, IQR: 24-60). Family specific

4 two-point logarithm of the odds (LOD) score was 3.96 using a dominant model and $80 \%$ penetrance,

5 which strongly supports the linkage between phenotype and variant (44). Following this finding, we

6 approached other European inherited cardiac disease units for genetically sequenced DCM patients

7 with missense variants in Cys3575. An additional Danish DCM family captured our attention. In this

8 family the same Cys3575 is mutated but in this case Cys is replaced by Arg (g.178741559A>G).

9 Cosegregation analysis in this smaller family was limited as only two individuals showed DCM

10 (Figure 7C). The proband was a young female with a very low LVEF (20\%) and a severely dilated left

11 ventricle $(\mathrm{LVEDD}=75 \mathrm{~mm})$ who underwent heart transplantation at 17 years of age. Her father was

12 diagnosed with DCM when he was 54 years old and died at the age of 73 years. Genetic analyses of

13 his kindred showed that the father was an obligate carrier of the p.Cys3575Arg variant supporting the

14 pathogenic nature of the variant. The 52-year-old proband's half-sister who also carries the variant had normal cardiac phenotype but a borderline depressed global longitudinal strain of $-17 \%$.

17 To provide functional evidence that the variants are pathogenic, we undertook cellular and molecular phenotyping studies. Human-induced-pluripotent-stem-cell (hiPSC)-derived cardiomyocytes carrying the p.Cys3575Ser variant in homozygosity show deficient contractility (Figure 7D), further supporting pathogenicity (45). Similarly to previous results (45), we did not detect any functional deficit in

21 heterozygous p.Cys3575Ser cells (Supplementary Figure S5B). At the DNA level, the mutation lies

22 in exon 49 of titin, more than 100 bp away from exon-exon junctions. Therefore, it is highly unlikely

23 that the mutations interfere with native mRNA splicing sites, and the probability of inducing new 24 splicing sites is also very low according to bioinformatics predictions (Supplementary Figure S5C,D).

25 Using far-UV circular dichroism, we observed that a recombinant version of I21 Cys3575Ser domain

26 (Supplementary Note S2, Supplementary Figure S5E) preserves the overall wild-type fold (Figure 
1 7E) but is barely stable at physiological temperatures (melting temperature, $\mathrm{T}_{\mathrm{m}}=38 \pm 1^{\circ} \mathrm{C} v s 55 \pm 1^{\circ} \mathrm{C}$

2 for wild-type domain, errors are SD of the sigmoidal fit, Figure 7F, Supplementary Figure S5F).

3 Given the highly dissimilar physicochemical properties of Arg and Cys, we expect the Cys3575Arg

4 mutation to lead also to strong domain destabilization.

6 Our MS results show that Cys3575 is oxidized in cardiac titin. The parent I21 domain contains a second

7 highly conserved cysteine (residue 3586 in human titin), which is located in strand $\mathrm{G}$ at a distance that

8 is compatible with disulfide bond formation with Cys3575 (21) (Figure 7B). The MS data were

9 inconclusive with regards to the oxidation state of Cys3586 since $\mathrm{mBBr}$-derivatized peptides were

10 found for this position also in -DTT control samples. No mBBr-modified peptides for the equivalent

11 Cys3592 in P0 mouse titin were detected. In both DCM variants targeting Cys3575, the disulfide

12 between positions 3575 and 3586 cannot be established, which would leave the mutant domains in a

13 highly destabilized state according to our circular dichroism data (Figure 7F).

In summary, our results show that Cys3575, which we demonstrate to be oxidized in human myocardium, is a target of DCM mutations that destabilize the parent domain and alter the mechanical function of hiPSC-derived cardiomyocytes.

\section{DISCUSSION}

The high nucleophilicity of the thiol side chain makes cysteine the most reactive protein amino acid.

21 In addition, cysteine residues are sensitive to irreversible oxidation, which can result in protein

22 aggregation and degradation (46). As a consequence, proteins tend to include cysteine only if the

23 associated functional benefits compensate the risks stemming from its peculiar physicochemical

24 properties. Indeed, cysteine is among the least frequent but most conserved residues in proteins (47),

25 reflecting key functional roles including configuration of enzyme active sites or metal chelation sites

26 (48, 49), establishment of disulfide bonds (41), deployment of electron transport systems $(50)$, or as 
redox sensors (51). Although several in vitro experiments over the last years have shown that cysteine

2 oxidation is a strong modulator of titin mechanics, evidence that such modulation is functionally

3 relevant in vivo has been lacking (18).

5 In vitro, conserved cysteines $\mathrm{B}, \mathrm{F}$ and $\mathrm{G}$ can be induced to form disulfide bonds that alter the

6 nanomechanics of the parent Ig domains $(21,24)$. Hence, a plausible scenario is that equivalent

7 disulfides are present naturally in native titin as mechanical rheostats. This view has been traditionally

8 considered unlikely taking into consideration the reducing environment of the cytosol in which

9 sarcomeres are located $(23,31)$. However, our biochemical and MS results strongly support the

10 existence of disulfides in titin in basal conditions in both human and mice (Figure 2-5), which adds to

11 the increasing pool of evidence that redox compartmentalization of cardiomyocytes is complex (52).

12 In this regard, future research will aim at identifying the biochemical systems responsible for disulfide

13 formation in titin (53-55), and whether equivalent constitutive oxidations target other proteins in the

14 sarcomere $(21,56-58)$.

Our results indicate that titin I-band cysteines that cannot establish disulfide bonds, such as conserved

17 Cys47, can also be oxidized in basal conditions. By integrating current knowledge on the mechanical

18 effects of cysteine oxidations obtained at the single-molecule level, our Monte Carlo simulations have 19 allowed us to explore the range of oxidative mechanical modulation of titin (Figure 6). The simulations

20 suggest that the extent and direction of mechanical modulation depends on the specific titin isoform,

21 the biochemical nature of the oxidative modification (disulfide $v s$ S-thiolation) and the range of forces

22 experienced by titin. Two observations stemming from the Monte Carlo simulations are relevant for

23 the redox modulation of titin mechanics at physiologically relevant forces, which are generally assumed

24 to be $<10 \mathrm{pN} /$ titin molecule $(19,59-61)$. First, due mainly to increased Ig unfolding rates, oxidations

25 render the titin filament much more dynamic, up to one order of magnitude at low forces (Figure

$26 \mathbf{6 F}, \mathbf{H})$. More frequent Ig unfolding can change the landscape of interactors, modulating titin-based 
1 mechanosignaling (42), whereas high folding rates enabled by disulfide bonds can sustain titin's

2 contribution to active muscle contraction $(33,59,62)$. Monte Carlo simulations also illustrate that

3 disulfides can result both in stiffening or softening of titin (Figure 6E,G), a consequence of the

4 opposing effects of disulfides on contour length and the (un)folding rates of Ig domains. This

5 observation, together with the softening effects of S-thiolation, can reconcile seemingly contradictory

6 results of experiments using muscle preparations. For instance, treatment of human cardiomyocytes

7 with the reducing enzyme thioredoxin results in drops in passive tension during oscillatory changes in

8 length (23) while incubation with DTT results in increased passive tension during stepwise length

9 increases (22). Similar DTT incubations also increase passive tension of rat and human skeletal fibers

10 (30). Regarding oxidative modifications, treatment of mouse cardiac muscle with $\mathrm{H}_{2} \mathrm{O}_{2}$ induces

11 increased passive tension in oscillatory protocols (29), whereas specific S-thiolation reactions lead to

12 softening during stepwise extension of human cardiomyocytes (22), an effect also observed in human

13 and rat skeletal muscle (30).

Overall, the Monte Carlo simulations show that the range of mechanical modulation of titin achieved by redox posttranslational modifications is ample. We have shown that right after birth, cardiac titin

17 becomes more oxidized (Figure 3). We speculate that oxidation can allow titin to rapidly fulfil more demanding ex utero mechanical functions (63), even before the isoform content completes transition to an adult profile over a longer timescale of days $(4,38)$ (Supplementary Figure S3B). While under these physiological conditions the boost of titin oxidation is beneficial, we speculate that altered redox

21 signaling may cause titin dysfunction during disease (57), for instance contributing to myocardial 22 stiffening following myocardial infarction (64).

24 Our functional and conservation analyses show that cysteines are important for the modulation of titin 25 mechanics, narrowing screening efforts to identify missense mutations that cause DCM. As a 26 consequence, we were able to demonstrate that alteration of the conserved Cys3575, a residue which 
1 we detect to be oxidized in cardiac titin, is responsible for DCM in humans (Figure 7). Moreover, from

2 a mechanistic point of view, we have detected extensive domain destabilization of the Cys3575Ser

3 mutant, which may trigger excessive titin degradation and similar downstream effects as in the case of

4 titin truncating variants. We have recently demonstrated a similar scenario in missense mutations of

5 cardiac myosin-binding protein $\mathrm{C}$ that cause hypertrophic cardiomyopathy (65). We speculate that

6 given the important role of cysteines in modulating titin mechanics, the fold of titin Ig domains may

7 have evolved to accommodate these residues, and that missense mutations targeting conserved

8 cysteines may result in strong destabilization and lead to disease.

9

The notion that missense mutations in titin could cause DCM is not new; however, our study is the first to unequivocally confirm that TTN missense variants can cause DCM in humans. In 2002, Gerull et al. reported that the missense mutation Trp976Arg in Ig domain 3 of titin (dbSNP: rs267607155), which was found in a moderately big DCM family, could cause the disease (16). The calculated LOD score for this family is 2.73 assuming an $80 \%$ penetrance of the disease, which is below the value of 3 usually considered to be associated with causal mutations (44). Interestingly, in our alignment, Trp976 is 100\% evolutionary conserved along 35 species, and it is also 99\% structurally conserved in human and mouse Ig domains highlighting its potential role in Ig domain stabilization (16). Since the identification of Trp976Arg, there have been anecdotal reports suggesting that $T T N$ missense variants can be indeed pathogenic $(10,66)$. A spontaneous canine DCM model caused by a missense variant has been reported. This model exhibited a TTN missense variant in the Ig-like domain I71 in the I-band of the cardiac N2BA isoform that was significantly associated with DCM $(\mathrm{p}<0.0001)$ in a family of Doberman pinschers. This variant corresponds to p.Gly8898Arg (ENST00000589042.5) in humans and it affects a 100\% conserved glycine in our alignment (67). In humans, a 2015 paper reported four small DCM kindreds with 5 missense rare variants in TTN considered "severe" according to bioinformatic tools (68). As there were only 2 DCM individuals genotyped per family in the study, the evidence of pathogenicity was weak due to limited cosegregation and the case for TTN missense variants as definite 
1 cause of DCM remained open. Rare missense variants in TTN are extremely frequent hampering

2 functional classification when interpreting NGS studies. This is exemplified by a recent study showing 3 that in 530 DCM patients, almost 7\% of them had rare TTN missense variants predicted to be deleterious 4 by bioinformatics filtering. When compared to a large reference population database (ExAC) there was 5 not a significant enrichment of the rare missense variants in the DCM population compared with ExAC 6 and authors concluded that TTN missense variants should be classified as likely benign in the clinical 7 diagnostic workflow (17). Our results confront with this conclusion and have direct application into 8 clinical practice. Currently, rare TTN missense variants found in DCM genetic studies are considered 9 unknown significance or likely benign and no further action is pursued. Based on our demonstration of 10 the important biological role of titin cysteines and that missense mutations can cause DCM, now if a 11 rare missense variant affecting a conserved cysteine in TTN is identified, predictive genetic testing 12 might be performed. In these cases, functional assays and evaluation of the variant in relatives to 13 confirm cosegregation should be undertaken. Similar strategies could be put in place in the future for other evolutionary and structurally conserved residues in titin.

\section{METHODS}

\section{Human subject research}

18 Human subject research was carried out in accordance with principles outlined in the Declaration of Helsinki. Probands with DCM were systematically studied by NGS with a panel of 121 genes associated or possibly associated with DCM at a certified clinical laboratory. These analyses did not

21 show any pathogenic/likely pathogenic rare variants. Relatives were genetically studied for TTN

22 missense variants found in probands by Sanger sequencing. Additionally, three phenotype-positive

23 distant relatives of the family with the p.Cys3575Ser variant underwent exome sequencing that did not

24 reveal commonly shared additional rare variants in cardiomyopathy-associated genes. Cardiac 25 evaluation of all subjects included ECG and echocardiography. Selected individuals underwent cardiac 26 magnetic resonance and additional cardiac tests according to clinical practice. DCM was defined as a 
1 left ventricular ejection fraction $(\mathrm{LVEF})<50 \%(69)$. All individuals provided written consent. Study

2 was approved by Hospital Universitario Puerta de Hierro ethics committee. Procedures for procurement

3 of human left ventricular tissue from non-failing donor hearts that were not accepted for transplantation

4 were approved by the Ethical Committee of the Medical University of Graz (28-508 ex 15/16) and the

5 Instituto de Salud Carlos III (CEI PI 65_2017-v1). Upon ice-cold cardioplegia, cardiac biopsies were

6 harvested from the left ventricular free wall, quickly frozen in liquid nitrogen and stored at $-80^{\circ} \mathrm{C}$. Non-

7 failing hearts had echocardiographic evidence of preserved ejection fraction $(>50 \%)$ and a clinical

8 history that was free of cardiac abnormalities.

9 Animal research

10 All animals used in this work were CD1 mice housed and maintained in the animal facility at the CNIC

11 (Madrid, Spain) in accordance with national and European Legislation. Procedures were approved by

12 the CNIC Animal Welfare Ethics Committee and by the Area of Animal Protection of the Regional

13 Government of Madrid (PROEX 042/18).

14 Cysteine conservation analysis

15 The longest currently available titin sequences for the 37 species reported in (24) were aligned using

16 Clustal Omega, with the only exception of chicken, for which no titin sequence with enough coverage

17 was found in Uniprot, GenomeNet or NCBI databases. Sequence identification codes are listed in

18 Supplementary Note S3. Conservation values for all cysteine positions in the human titin sequence

19 (Uniprot Q8WZ42) were obtained as percentages of conservation not considering gaps in the

20 alignment. The vast majority of cysteine positions show very high occupancy ( $98 \%$ average occupancy,

21 Supplementary Figure S1), which supports robustness of the conservation analyses. The alignment

22 and homology models of titin Ig domains of human titin have been published before (21); alignment

23 of mouse titin Ig domains appearing in Uniprot was obtained similarly using Clustal Omega

24 (Supplementary Note S4). These Ig domain alignments were used to classify titin cysteines for

25 structural conservation and mass spectrometry analyses.

\section{In-gel determination of reversibly oxidized thiols}


1 Our protocol for in-gel determination of reversibly oxidized thiols was adapted and optimized from

2 previous reports $(24,32)$. Protein extracts were obtained by cryopulverization of myocardial tissue

3 followed by homogenization in sample buffer (50 mM Tris-HCl, $10 \mathrm{mM}$ EDTA, 3\% SDS, pH 6.8; 40

$4 \quad \mu \mathrm{l}$ per mg of tissue) containing $50 \mathrm{mM}$ NEM, unless indicated otherwise. Samples were run on SDS-

5 PAGE gels (3.5\% for analysis of titin, $12 \%$ for other proteins), and oxidized thiols were then reduced

6 by incubation of the gel with $10 \mathrm{mM}$ DL-dithiothreitol (DTT) (Sigma-Aldrich) in $50 \mathrm{mM}$ ammonium

7 bicarbonate, $\mathrm{pH} 8.8$ at $60^{\circ} \mathrm{C}$. After 3 washes of 20 min with sample buffer, the newly reduced thiols

8 were labeled by incubation with $5 \mathrm{mM} \mathrm{mBBr}$ (Merk Millipore) in sample buffer during $2 \mathrm{~h}$ at room

9 temperature in the dark. The excess of $\mathrm{mBBr}$ was removed by three washes with destaining solution

10 (40\% ethanol, 10\% acetic acid) lasting $1 \mathrm{~h}$, overnight and again $1 \mathrm{~h}$. Fluorescent bands were visualized

11 using a Gel-Doc (BioRad) with UV excitation (standard filters for ethidium bromide). Coomassie

12 staining of the same gel was used to normalize fluorescence signals. Quantification of the bands was

13 done by densitometry using Quantity One. Fluorescence signals coming from a replicate gel not treated

14 with DTT were subtracted. Building on a previous report (24), control samples prepared by mixing 15 different proportions of oxidized (I91-32/75) 8 polyprotein and its Cys-free version were also included

16 in the experiment in order to build calibration curves (Supplementary Figure S3). Two terminal

17 cysteines in the polyproteins were not considered in these calculations since their oxidation status could

18 not be ascertained. When comparing oxidation of different proteins, we normalized the oxidation

19 signals by their density of cysteines (Uniprot entries Q8WZ42-1 and -3 for human N2BA and N2B

20 respectively, A2ASS6-1 and -2 for mouse N2BA and N2B respectively, and Q02566 for mouse cardiac

21 myosin).

\section{Mass spectrometry}

23 Titin bands were sliced from SDS-PAGE gels used for fluorescence quantifications, diced and washed 24 by incubation with $200 \mu \mathrm{l}$ high purity water (Fluka CHROMASOLVTM LC-MS) for 10 min at 1200 $25 \mathrm{rpm}$ (4 times). The resulting gel pieces were dehydrated by 2 incubations with $100 \%$ acetonitrile and 1 26 incubation with $50 \mathrm{mM}$ ammonium bicarbonate (pH 8.8) in acetonitrile (both incubations, $15 \mathrm{~min}$ 
1 shaking at $1200 \mathrm{rpm})$. After drying using a SpeedVac, the gel pieces were incubated with modified

2 trypsin from porcine pancreas (Sigma Aldrich) in digestion buffer (10\% acetonitrile in $50 \mathrm{mM}$

3 ammonium bicarbonate, $\mathrm{pH} 8.8$ ) for $2 \mathrm{~h}$ in ice to allow the diffusion of the inactive enzyme, and then

4 at $37^{\circ} \mathrm{C}$ overnight. Digested tryptic peptides were extracted with $1 \%$ trifluoroacetic acid in acetonitrile

5 (15 min incubation, 1200 rpm shaking) and dried. Finally, they were resuspended in $1 \%$ trifluoroacetic

6 acid by pulse-vortexing and sonication, and desalted using OMIX commercial columns (Biomaster

7 group). Peptides were then injected into a reversed phase C-18 nano-column (Acclaim PepMap RSLC,

$875 \mu \mathrm{m}$ internal diameter and $50 \mathrm{~cm}$ length), and eluted to be analysed in a hybrid quadrupole-Orbitrap

9 Q Exactive mass spectrometer (Thermo Scientific) for protein identification. A continuous acetonitrile

10 gradient consisting of $0-30 \%$ A for $120 \mathrm{~min}, 50-90 \% \mathrm{~B}$ for $3 \mathrm{~min}(\mathrm{~A}=0.1 \%$ formic acid; $\mathrm{B}=98 \%$

11 acetonitrile, $0.1 \%$ formic acid) at a flow rate of $200 \mathrm{~nL} / \mathrm{min}$ was used to elute tryptic peptides from the nano-column to a nanospray emitter for real time ionization and induced fragmentation. High resolution mass spectra were acquired in a data-dependent manner with dynamic exclusion by combining a MS spectrum (from 400-1500 m/z, 120,000 resolution) followed by the MS/MS spectra (60,000 resolution) from the 15 most intense species. For protein identification, tandem mass spectra were extracted and charge state was deconvoluted by Proteome Discoverer 1.4.0.288 (Thermo Fisher Scientific). All MS/MS spectra were analyzed using SEQUEST (Thermo Fisher Scientific). Full proteome search databases (mouse: UniProtKB/Swiss-Prot April27_2016, 48736 sequences; human: UniProtKB/SwissProt, November 2019, 74333 sequences) were supplemented with 116 cRAP proteins (common

21 missed cleavages and used $20 \mathrm{ppm}$ and $20 \mathrm{mDa}$ precursor and fragment mass tolerances, respectively.

22 NEM- or mBBr-modified cysteines, and oxidation of methionine were specified as variable 23 modifications. In these initial searches, a target-decoy fixed value PSM validation strategy was used to 24 filter peptides according to XCorr values (more than 1.2 in doubly-charged peptides, or more than 1.4 25 in triply-charged peptides for $<5 \%$ FDR, and more than 1.42 in doubly-charged peptides, or more than 261.79 for triply-charged peptides for $<1 \%$ FDR). Since the vast majority of the identified peptides 
1 belonged to titin in searches against full proteome databases, to optimize coverage of cysteine peptides

2 we repeated the search using titin-only reduced search databases (39). Peptide identifications were

3 validated using Vseq, an in-house developed tool that evaluates the mass tolerance, intensity,

4 fragmentation goodness and quantitative value of MS/MS scans (40). Maximum matched ions value

5 for a sequence was calculated considering the number of residues multiplied by 2 , to cover the complete

6 theoretical length of the main fragmentation series B and Y. Since experiments were acquired using a

7 label-free strategy and using HCD for ion-induced dissociation, we set a threshold of $25 \%$ minimum

8 matched ions, which corresponds to at least $50 \%$ of the Y-HCD-enhanced fragmentation series. We

9 also filtered peptide identifications according to their E-scores, which is defined as the dot product of

10 the intensities for all matched ions regardless of their charge state. We applied an E-score threshold of

$11>0.01$. We verified that none of the unidentified scans showing a very low XCorr and matched ions

12 value was above this limit. To estimate final FDR values, we repeated the searches considering the

13 same parameters against the corresponding decoy databases. FDR was below $2 \%$ in all cases. To

14 minimize false positive detection of oxidized cysteines, we analyzed in parallel the same samples

15 without including DTT in the derivatization reactions. For each sample, cysteine positions belonging

16 to mBBr-peptides identified both in the -DTT and +DTT treated specimens were excluded from the

17 analysis $(27 \pm 5 \%$ and $30 \pm 4 \%$ of the oxidized cysteine-containing peptides identified in mouse and in

18 human). OS were calculated for each cysteine following

$$
\text { OS }=\frac{\# \text { Ox }-\# \text { Red }}{\# \text { Samples }}
$$

22 where \#Ox and \#Red are the number of samples in which the cysteine is detected as always oxidized

23 or reduced, respectively, and \#Samples is the total number of samples in which the cysteine is detected,

24 including those instances in which both the oxidized and reduced forms are detected. OS ranges from

$25-1$ to 1 ; these extreme values are given to cysteines that are always detected as reduced or oxidized, 
1 respectively.

\section{Monte Carlo simulations}

3 We constructed a simplified model of titin's I band to predict the length of the molecule under force

4 upon redox modifications using Monte Carlo simulations $(21,22)$. Simulations calculate the length of

5 the two entropic regions of the protein (PEVK and N2Bus) and the Ig domains, according to the Freely

6 Jointed Chain model of polymer elasticity and the Bell's model of force-dependent reactions

7 (Supplementary Note S1). The length of the entropic regions and the number of Ig domains in each

8 isoform were determined according to human titin sequence in Uniprot, further curated in (21). Titin

9 domains were classified according to their cysteine content, which was used to determine the

10 modifications they could be target of (Supplementary Table S1) and their mechanical and kinetic

11 parameters (Supplementary tables S2-S5). The constructed model was subject to an oscillating force

12 protocol (triangular waves, $1 \mathrm{~Hz}, 10 \mathrm{~ms}$ simulation step) for 3 hours. Some simulations were also

13 conducted using $1 \mathrm{~ms}$ simulation steps, leading to equivalent results. In the initial state all domains are

14 folded. If oxidative modifications are present, they occur at all potential target sites. In conditions

15 involving disulfide bonds, all domains capable of disulfide isomerization contain the BG disulfide in

16 the initial state (21). We averaged 10 independent simulations for each condition. The code for the

17 simulations and the subsequent analysis of the data was conducted in Igor Pro.

\section{hiPSC-derived cardiomyocytes characterization}

$19 T^{\text {TTN }}{ }^{\text {Cys375Ser }}$-hiPSC lines were generated by using the CRISPR/Cas9 technology to introduce the 20 corresponding point mutation in the TTN allele of a wild-type (WT) hiPSC line (HDF-iPS-SV10 21 Spanish National Stem Cell Bank, ISCIII). The crRNA 5'-TGTATGGCCAGTAATGACTA-3', was 22 designed by the online CRISPOR-TEFOR software (http://crispor.tefor.net/crispor.py), matching the 23 nucleotide involved in the mutation as the first base of sequence. The ssODN sequence lies in the No- 


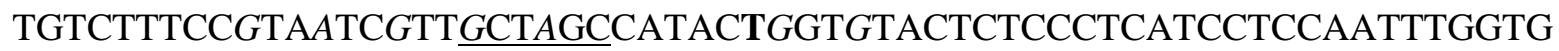
AACAGAATGATCAGGCTATG, and was designed by inserting the intended mutation (bold), as well as silent variants (italic) to avoid unwanted cutting of the ribonucleoprotein complex (RPN) and to allow genotyping by restriction enzyme digestion (NheI-underlined). The primers used to genotype selected clones were hTTN-Fwd: 5'-CAGGTGGACTCACCCAACAT-3' and hTTN-Rev 5'CCAGGAATAAAGCAGAAGAAGGC- 3'. Synthetic crRNA and tracrRNA (AltR CRISPRCas9 system, IDT) were mixed in equimolar concentrations (22 pmol/each), combined with $25 \mu \mathrm{M}$ Cas9 Nuclease 3NLS to form the ribonucleoprotein complex. The RNP complex plus ssODN and AltR Cas9 Electroporator Enhancer (22 pmol/each) were electroporated, and cells were recovered and split into p100 dishes as described (70). After 7-10 days, colonies were picked up and split into p96 and p24 well plates for screening and maintenance respectively. DNA from 200 clones was isolated, PCR amplified and NheI digested. Several clones were identified as positive and Sanger sequenced. One proved to be homozygous and another one heterozygous for the desired mutation. For cardiac differentiation, WT and $T T N^{\text {Cys3575Ser }}$-hiPSC lines were expanded and dissociated into single cells as described (70). Briefly cells were seeded at a density of $1-1.2 \times 10^{6}$ cells per well in Essential E8 medium supplemented with $10 \mu \mathrm{M}$ ROCK inhibitor. Upon achieving confluence, they were treated with $8 \mu \mathrm{M}$ GSK3 inhibitor (CHIR99021, Stemgent) in RPMI supplemented with B27 lacking insulin (Thermo Fisher). 24 hrs later, the medium was changed to RPMI/B27-insulin. On day 3, cells were treated with 5 $\mu \mathrm{M}$ Wnt inhibitor IWP4 (Stemgent) in RPMI/B27 minus insulin medium, which was replaced two days later. From day 7 onwards, differentiated cells were maintained in RPMI/B27 medium supplemented with insulin. Beating areas were visible between days 8-10 from the onset of the differentiation. For iPSC-derived cardiac monolayer maturation, cells were disaggregated at day 32 by incubation with $0.25 \%$ trypsinEDTA for $10 \mathrm{~min}$ at $37^{\circ} \mathrm{C}$ and seeded onto matrigel-coated PDMS membranes (71) at a density of 1.25 $\mathrm{x} 10^{5}$ cells/well in 12-well plates. WT and TTN ${ }^{\text {Cys3575Ser }}$-hiPSC derived cardiomyocytes (hiPSC-CMs) were maintained in RPMI/B27 medium supplemented with insulin for 1 week for further maturation. 
1 Video recording was taken and analyzed for single cell contraction amplitude using the Musclemotion

$2 \operatorname{algorithm~(72).~}$

\section{Protein purification}

4 The E. coli-codon-optimized-cDNA coding for I21 WT was ordered to GeneArt and the Cys3575Ser

5 mutant was produced by PCR-mutagenesis (sequences available in Supplementary Note S2). cDNAs

6 were cloned in a custom-modified pQE80 expression plasmid (Qiagen) using BamHI and BgIII

7 restriction enzymes. Final expression plasmids were verified by Sanger sequencing. Domains were

8 expressed in E. coli BLR(DE3). For the I21 WT domain cultures at $\mathrm{OD}_{600}=0.6-1$ were induced with

$91 \mathrm{mM}$ isopropyl $\beta$-D-1-thiogalactopyranoside (IPTG) and incubated for $3 \mathrm{~h}$ at $37^{\circ} \mathrm{C}$. For the

10 Cys3575Ser mutant, the culture was induced with $0.4 \mathrm{mM}$ IPTG and incubated at $16^{\circ} \mathrm{C}$

11 overnight. Purification of His-tagged domains was achieved by metal affinity (using $1 \mathrm{mM}$ DTT

12 containing buffers) and gel filtration chromatographies following published protocols (73). Proteins

13 were eluted from the final size-exclusion chromatography in $20 \mathrm{mM} \mathrm{NaPi}, \mathrm{pH}$ 6.5, $63.6 \mathrm{mM} \mathrm{NaCl}$ and

14 stored at $4^{\circ} \mathrm{C}$. Purity of the preparations was evaluated by SDS-PAGE (Supplementary Figure S5E).

\section{Circular dichroism}

16 CD spectra were collected using a Jasco J-810 spectropolarimeter. Purified proteins were tested in 20

$17 \mathrm{mM} \mathrm{NaPi}, \mathrm{pH} 6.5$ and $63.6 \mathrm{mM} \mathrm{NaCl}$ at $0.3 \mathrm{mg} / \mathrm{ml}$ protein concentration in 0.1 -cm-pathlength quartz

18 cuvettes. Protein concentration was obtained from $A_{280}$ values using theoretical extinction coefficients

19 estimated using ProtParam $(74)\left(E^{0.1 \%}=0.925\right)$. Spectra were recorded at $50 \mathrm{~nm} / \mathrm{min}$ scanning speed

20 and a data pitch of $0.2 \mathrm{~nm}$. Four scans were averaged to obtain the final spectra. The contribution of

21 the buffer was subtracted and spectra were normalized by peptide bond concentration. To study thermal

22 denaturation, $\mathrm{CD}$ signal at $215 \mathrm{~nm}$ was monitored as temperature increased from 25 to $85{ }^{\circ} \mathrm{C}$ at a rate

23 of $30^{\circ} \mathrm{C} / \mathrm{h}$. Temperature control was achieved using a Peltier thermoelectric system. To estimate $\mathrm{T}_{\mathrm{m}}$,

24 changes in CD signal were fit to a sigmoidal function using IGOR Pro (Wavemetrics).

\section{Statistics}


1 Statistical tests were run using Graph Pad Prism 8.3.1 for Windows. To evaluate the tendency of

2 different categories of cysteines to be oxidized ( $O S>0)$, we estimated the corresponding p-values using

3 the hypergeometric distribution function implemented in Excel (Microsoft). Errors are given by SEM,

4 unless indicated otherwise.

\section{$5 \quad$ References and Notes}

6 1. J. K. Freundt, W. A. Linke, Titin as a force-generating muscle protein under regulatory

7 control. J Appl Physiol (1985) 126, 1474-1482 (2019).

8 2. B. R. Anderson, H. L. Granzier, Titin-based tension in the cardiac sarcomere: Molecular

$9 \quad$ origin and physiological adaptations. Progress in Biophysics and Molecular Biology 110, 204-

$217(2012)$.

11 3. E. C. Eckels, R. Tapia-Rojo, J. A. Rivas-Pardo, J. M. Fernandez, The Work of Titin Protein

12 Folding as a Major Driver in Muscle Contraction. Annual review of physiology 80, 327-351

13 (2018).

14 4. A. Opitz Christiane, C. Leake Mark, I. Makarenko, V. Benes, A. Linke Wolfgang,

15 Developmentally Regulated Switching of Titin Size Alters Myofibrillar Stiffness in the

16 Perinatal Heart. Circulation Research 94, 967-975 (2004).

17 5. A. Freiburg, K. Trombitas, W. Hell, O. Cazorla, F. Fougerousse, T. Centner, B. Kolmerer, C.

18 Witt, J. S. Beckmann, C. C. Gregorio, H. Granzier, S. Labeit, Series of exon-skipping events in

19 the elastic spring region of titin as the structural basis for myofibrillar elastic diversity.

$20 \quad$ Circulation research 86, 1114-1121 (2000).

21 6. M. Kruger, in Cardiac Cytoarchitecture, E. Ehler, Ed. (Springer International Publishing, 22 2015), pp. 109-124.

23 7. D. S. Herman, L. Lam, M. R. G. Taylor, L. B. Wang, P. Teekakirikul, D. Christodoulou, L. 24 Conner, S. R. DePalma, B. McDonough, E. Sparks, D. L. Teodorescu, A. L. Cirino, N. R. Banner, 
D. J. Pennell, S. Graw, M. Merlo, A. Di Lenarda, G. Sinagra, J. M. Bos, M. J. Ackerman, R. N. Mitchell, C. E. Murry, N. K. Lakdawala, C. Y. Ho, P. J. R. Barton, S. A. Cook, L. Mestroni, J. G. Seidman, C. E. Seidman, Truncations of Titin Causing Dilated Cardiomyopathy. New Engl J Med 366, 619-628 (2012).

8. D. Kellermayer, J. E. Smith, H. Granzier, Titin mutations and muscle disease. Pflügers Archiv European Journal of Physiology 471, 673-682 (2019).

9. D. Fatkin, I. G. Huttner, J. C. Kovacic, J. G. Seidman, C. E. Seidman, Precision Medicine in the Management of Dilated Cardiomyopathy. Journal of the American College of Cardiology 74, 2921 (2019).

10. J. S. Ware, S. A. Cook, Role of titin in cardiomyopathy: from DNA variants to patient stratification. Nature Reviews Cardiology 15, 241-252 (2018).

11. S. Cuenca, M. J. Ruiz-Cano, J. R. Gimeno-Blanes, A. Jurado, C. Salas, I. Gomez-Diaz, L. PadronBarthe, J. J. Grillo, C. Vilches, J. Segovia, D. Pascual-Figal, E. Lara-Pezzi, L. Monserrat, L. Alonso-Pulpon, P. Garcia-Pavia, Genetic basis of familial dilated cardiomyopathy patients undergoing heart transplantation. The Journal of Heart and Lung Transplantation 35, 625635 (2016).

12. S. van Heesch, F. Witte, V. Schneider-Lunitz, J. F. Schulz, E. Adami, A. B. Faber, M. Kirchner, H. Maatz, S. Blachut, C. L. Sandmann, M. Kanda, C. L. Worth, S. Schafer, L. Calviello, R. Merriott, G. Patone, O. Hummel, E. Wyler, B. Obermayer, M. B. Mucke, E. L. Lindberg, F. Trnka, S. Memczak, M. Schilling, L. E. Felkin, P. J. R. Barton, N. M. Quaife, K. Vanezis, S. Diecke, M. Mukai, N. Mah, S. J. Oh, A. Kurtz, C. Schramm, D. Schwinge, M. Sebode, M. Harakalova, F. W. Asselbergs, A. Vink, R. A. de Weger, S. Viswanathan, A. A. Widjaja, A. Gartner-Rommel, H. Milting, C. Dos Remedios, C. Knosalla, P. Mertins, M. Landthaler, M. 
Vingron, W. A. Linke, J. G. Seidman, C. E. Seidman, N. Rajewsky, U. Ohler, S. A. Cook, N. Hubner, The Translational Landscape of the Human Heart. Cell 178, 242-260 e229 (2019).

13. A. M. Roberts, J. S. Ware, D. S. Herman, S. Schafer, J. Baksi, A. G. Bick, R. J. Buchan, R. Walsh, S. John, S. Wilkinson, F. Mazzarotto, L. E. Felkin, S. Gong, J. A. MacArthur, F. Cunningham, J. Flannick, S. B. Gabriel, D. M. Altshuler, P. S. Macdonald, M. Heinig, A. M. Keogh, C. S. Hayward, N. R. Banner, D. J. Pennell, D. P. O'Regan, T. R. San, A. de Marvao, T. J. Dawes, A. Gulati, E. J. Birks, M. H. Yacoub, M. Radke, M. Gotthardt, J. G. Wilson, C. J. O'Donnell, S. K. Prasad, P. J. Barton, D. Fatkin, N. Hubner, J. G. Seidman, C. E. Seidman, S. A. Cook, Integrated allelic, transcriptional, and phenomic dissection of the cardiac effects of titin truncations in health and disease. Science translational medicine 7, 270ra276 (2015).

14. C. A. Tharp, M. E. Haywood, O. Sbaizero, M. R. G. Taylor, L. Mestroni, The Giant Protein Titin's Role in Cardiomyopathy: Genetic, Transcriptional, and Post-translational Modifications of TTN and Their Contribution to Cardiac Disease. Frontiers in Physiology 10, (2019).

15. M. Gigli, R. L. Begay, G. Morea, S. L. Graw, G. Sinagra, M. R. G. Taylor, H. Granzier, L. Mestroni, A Review of the Giant Protein Titin in Clinical Molecular Diagnostics of Cardiomyopathies. Frontiers in Cardiovascular Medicine 3, (2016).

16. B. Gerull, M. Gramlich, J. Atherton, M. McNabb, K. Trombitas, S. Sasse-Klaassen, J. G. Seidman, C. Seidman, H. Granzier, S. Labeit, M. Frenneaux, L. Thierfelder, Mutations of TTN, encoding the giant muscle filament titin, cause familial dilated cardiomyopathy. Nat Genet 30, 201-204 (2002).

17. O. Akinrinade, T. Heliö, R. H. Lekanne Deprez, J. D. H. Jongbloed, L. G. Boven, M. P. van den Berg, Y. M. Pinto, T.-P. Alastalo, S. Myllykangas, K. v. Spaendonck-Zwarts, J. P. v. Tintelen, P. 
A. van der Zwaag, J. Koskenvuo, Relevance of Titin Missense and Non-Frameshifting Insertions/Deletions Variants in Dilated Cardiomyopathy. Scientific Reports 9, 4093 (2019).

18. E. Herrero-Galán, I. Martínez-Martín, J. Alegre-Cebollada, Redox regulation of protein nanomechanics in health and disease: Lessons from titin. Redox Biology 21, 101074 (2019).

19. H. Li, W. A. Linke, A. F. Oberhauser, M. Carrion-Vazquez, J. G. Kerkvliet, H. Lu, P. E. Marszalek, J. M. Fernandez, Reverse engineering of the giant muscle protein titin. Nature 418, 998-1002 (2002).

20. M. S. Kellermayer, L. Grama, Stretching and visualizing titin molecules: combining structure, dynamics and mechanics. J Muscle Res Cell Motil 23, 499-511 (2002).

21. D. Giganti, K. Yan, C. L. Badilla, J. M. Fernandez, J. Alegre-Cebollada, Disulfide isomerization reactions in titin immunoglobulin domains enable a mode of protein elasticity. Nature communications 9, 185 (2018).

22. J. Alegre-Cebollada, P. Kosuri, D. Giganti, E. Eckels, J. A. Rivas-Pardo, N. Hamdani, C. M. Warren, R. J. Solaro, W. A. Linke, J. M. Fernandez, S-glutathionylation of cryptic cysteines enhances titin elasticity by blocking protein folding. Cell 156, 1235-1246 (2014).

23. A. Grutzner, S. Garcia-Manyes, S. Kotter, C. L. Badilla, J. M. Fernandez, W. A. Linke, Modulation of titin-based stiffness by disulfide bonding in the cardiac titin N2-B unique sequence. Biophys J 97, 825-834 (2009).

24. A. Manteca, J. Schonfelder, A. Alonso-Caballero, M. J. Fertin, N. Barruetabena, B. F. Faria, E. Herrero-Galan, J. Alegre-Cebollada, D. De Sancho, R. Perez-Jimenez, Mechanochemical evolution of the giant muscle protein titin as inferred from resurrected proteins. Nature structural \& molecular biology 24, 652-657 (2017). 
$125 . \quad$ B. S. Avner, K. M. Shioura, S. B. Scruggs, M. Grachoff, D. L. Geenen, D. L. Helseth, M. Farjah,

2 P. H. Goldspink, R. J. Solaro, Myocardial infarction in mice alters sarcomeric function via post-translational protein modification. Mol Cell Biochem 363, 203-215 (2012).

26. B. Bodi, E. P. Toth, L. Nagy, A. Toth, L. Martha, A. Kovacs, G. Balla, T. Kovacs, Z. Papp, Titin

27. J. Paulech, N. Solis, S. J. Cordwell, Characterization of reaction conditions providing rapid

28. H. Xiao, M. P. Jedrychowski, D. K. Schweppe, E. L. Huttlin, Q. Yu, D. E. Heppner, J. Li, J. Long,

29. J. Nedrud, S. Labeit, M. Gotthardt, H. Granzier, Mechanics on Myocardium Deficient in the N2B Region of Titin: The Cardiac-Unique Spring Element Improves Efficiency of the Cardiac Cycle. Biophysical Journal 101, 1385-1392 (2011). Research and Cell Motility, (2019).
O. Mayans, J. Wuerges, S. Canela, M. Gautel, M. Wilmanns, Structural evidence for a possible role of reversible disulphide bridge formation in the elasticity of the muscle protein titin. Structure 9, 331-340 (2001).

L. K. Rogers, B. L. Leinweber, C. V. Smith, Detection of reversible protein thiol modifications in tissues. Anal Biochem 358, 171-184 (2006). 
1 33. J. A. Rivas-Pardo, Y. Li, Z. Mártonfalvi, R. Tapia-Rojo, A. Unger, Á. Fernández-Trasancos, E. Herrero-Galán, D. Velázquez-Carreras, J. M. Fernández, W. A. Linke, J. Alegre-Cebollada, A HaloTag-TEV genetic cassette for mechanical phenotyping of proteins from tissues. Nature communications 11, 2060 (2020).

34. K. Wang, J. McClure, A. Tu, Titin: major myofibrillar components of striated muscle. Proceedings of the National Academy of Sciences 76, 3698 (1979).

35. R. E. Hansen, J. R. Winther, An introduction to methods for analyzing thiols and disulfides: Reactions, reagents, and practical considerations. Analytical Biochemistry 394, 147-158 (2009).

36. J. Vonderen, J. J. van Vonderen, A. A. W. Roest, M. L. Siew, F. J. Walther, S. B. Hooper, A. B. te Pas, Measuring Physiological Changes during the Transition to Life after Birth. Neonatology 105, 230-242 (2014).

37. B. N. Puente, W. Kimura, S. A. Muralidhar, J. Moon, J. F. Amatruda, K. L. Phelps, D. Grinsfelder, B. A. Rothermel, R. Chen, J. A. Garcia, C. X. Santos, S. Thet, E. Mori, M. T. Kinter, P. M. Rindler, S. Zacchigna, S. Mukherjee, D. J. Chen, A. I. Mahmoud, M. Giacca, P. S. Rabinovitch, A. Aroumougame, A. M. Shah, L. I. Szweda, H. A. Sadek, The oxygen-rich postnatal environment induces cardiomyocyte cell-cycle arrest through DNA damage response. Cell 157, 565-579 (2014).

38. S. Lahmers, Y. Wu, D. R. Call, S. Labeit, H. Granzier, Developmental control of titin isoform expression and passive stiffness in fetal and neonatal myocardium. Circulation research 94, 505-513 (2004).

39. W. S. Noble, Mass spectrometrists should search only for peptides they care about. Nature Methods 12, 605-608 (2015). 
1 40. S. Cogliati, E. Calvo, M. Loureiro, A. M. Guaras, R. Nieto-Arellano, C. Garcia-Poyatos, I.

2 Ezkurdia, N. Mercader, J. Vázquez, J. A. Enriquez, Mechanism of super-assembly of

3 respiratory complexes III and IV. Nature 539, 579-582 (2016).

4 41. P. Kosuri, J. Alegre-Cebollada, J. Feng, A. Kaplan, A. Ingles-Prieto, C. L. Badilla, B. R.

42. W. A. Linke, N. Hamdani, Gigantic business: titin properties and function through thick and thin. Circulation research 114, 1052-1068 (2014).

43. O. Cazorla, A. Freiburg, M. Helmes, T. Centner, M. McNabb, Y. Wu, K. Trombitas, S. Labeit, stiffness. Circ Res 86, 59-67 (2000).

45. J. T. Hinson, A. Chopra, N. Nafissi, W. J. Polacheck, C. C. Benson, S. Swist, J. Gorham, L. Yang, S. Schafer, C. C. Sheng, A. Haghighi, J. Homsy, N. Hubner, G. Church, S. A. Cook, W. A. Linke, C. S. Chen, J. G. Seidman, C. E. Seidman, HEART DISEASE. Titin mutations in iPS cells define sarcomere insufficiency as a cause of dilated cardiomyopathy. Science 349, 982-986 (2015). 46. M. Pajares, N. Jiménez-Moreno, I. H. K. Dias, B. Debelec, M. Vucetic, K. E. Fladmark, H. Basaga, S. Ribaric, I. Milisav, A. Cuadrado, Redox control of protein degradation. Redox Biology 6, 409-420 (2015). 
1 47. S. M. Marino, V. N. Gladyshev, Cysteine Function Governs Its Conservation and Degeneration and Restricts Its Utilization on Protein Surfaces. Journal of Molecular Biology 404, 902-916 (2010).

48. S. Verma, R. Dixit, K. C. Pandey, Cysteine Proteases: Modes of Activation and Future

49. N. M. Giles, A. B. Watts, G. I. Giles, F. H. Fry, J. A. Littlechild, C. Jacob, Metal and Redox

50. G. Bertoli, T. Simmen, T. Anelli, S. N. Molteni, R. Fesce, R. Sitia, Two Conserved Cysteine Triads in Human Ero1 $\alpha$ Cooperate for Efficient Disulfide Bond Formation in the Endoplasmic Reticulum. Journal of Biological Chemistry 279, 30047-30052 (2004). Becker, S. Nanadikar Maithily, B. Morgan, S. Dennerlein, M. Shah Ajay, A. El-Armouche, O. Nikolaev Viacheslav, M. Katschinski Dörthe, Redox Imaging Using Cardiac Myocyte-Specific Transgenic Biosensor Mice. Circulation Research 119, 1004-1016 (2016).

53. K. Kojer, J. Riemer, Balancing oxidative protein folding: The influences of reducing pathways on disulfide bond formation. Biochimica et Biophysica Acta (BBA) - Proteins and Proteomics $1844,1383-1390$ (2014).

54. J. Riemer, N. Bulleid, J. M. Herrmann, Disulfide formation in the ER and mitochondria: two solutions to a common process. Science 324, 1284-1287 (2009).

55. M. J. Saaranen, L. W. Ruddock, Disulfide Bond Formation in the Cytoplasm. Antioxidants \& Redox Signaling 19, 46-53 (2012). 
1 56. H. S. Chung, S. B. Wang, V. Venkatraman, C. I. Murray, J. E. Van Eyk, Cysteine oxidative posttranslational modifications: emerging regulation in the cardiovascular system. Circ Res 112, 382-392 (2013).

57. F. Cuello, I. Wittig, K. Lorenz, P. Eaton, Oxidation of cardiac myofilament proteins: Priming for dysfunction? Molecular Aspects of Medicine 63, 47-58 (2018).

58. C. X. C. Santos, N. Anilkumar, M. Zhang, A. C. Brewer, A. M. Shah, Redox signaling in cardiac myocytes. Free Radical Biology and Medicine 50, 777-793 (2011).

59. J. A. Rivas-Pardo, E. C. Eckels, I. Popa, P. Kosuri, W. A. Linke, J. M. Fernandez, Work Done by Titin Protein Folding Assists Muscle Contraction. Cell reports 14, 1339-1347 (2016).

60. A. Brynnel, Y. Hernandez, B. Kiss, J. Lindqvist, M. Adler, J. Kolb, R. van der Pijl, J. Gohlke, J. Strom, J. Smith, C. Ottenheijm, H. L. Granzier, Downsizing the molecular spring of the giant protein titin reveals that skeletal muscle titin determines passive stiffness and drives longitudinal hypertrophy. eLife 7, e40532 (2018).

61. W. A. Linke, M. R. Stockmeier, M. Ivemeyer, H. Hosser, P. Mundel, Characterizing titin's Iband Ig domain region as an entropic spring. Journal of Cell Science 111, 1567-1574 (1998).

62. E. C. Eckels, S. Haldar, R. Tapia-Rojo, J. A. Rivas-Pardo, J. M. Fernandez, The Mechanical Power of Titin Folding. Cell reports 27, 1836-1847 e1834 (2019).

63. Y.-Q. Zhou, F. S. Foster, R. Parkes, S. L. Adamson, Developmental changes in left and right ventricular diastolic filling patterns in mice. American Journal of Physiology-Heart and Circulatory Physiology 285, H1563-H1575 (2003).

64. S. Kötter, M. Kazmierowska, C. Andresen, K. Bottermann, M. Grandoch, S. Gorressen, A. Heinen, J. Moll, J. Scheller, A. Gödecke, J. W. Fischer, J. P. Schmitt, M. Krüger, Titin-Based Cardiac Myocyte Stiffening Contributes to Early Adaptive Ventricular Remodeling After Myocardial Infarction. Circulation Research, 1017-1029 (2016). 
65. C. Suay-Corredera, M. R. Pricolo, E. Herrero-Galan, D. Velazquez-Carreras, D. Sanchez-Ortiz, D. Garcia-Giustiniani, J. Delgado, J. J. Galano-Frutos, H. Garcia-Cebollada, S. Vilches, F. Dominguez, M. Sabater Molina, R. Barriales-Villa, G. Frisso, J. Sancho, L. Serrano, P. GarciaPavia, L. Monserrat, J. Alegre-Cebollada, Protein haploinsufficiency drivers identify MYBPC3 mutations that cause hypertrophic cardiomyopathy. medRxiv, 2020.2005.2004.20087726 (2020).

66. C. Chauveau, J. Rowell, A. Ferreiro, A Rising Titan: TTN Review and Mutation Update. Human mutation 35, 1046-1059 (2014).

67. K. M. Meurs, S. G. Friedenberg, J. Kolb, C. Saripalli, P. Tonino, K. Woodruff, N. J. Olby, B. W. Keene, D. B. Adin, O. L. Yost, T. C. DeFrancesco, S. Lahmers, S. Tou, G. D. Shelton, H. Granzier, A missense variant in the titin gene in Doberman pinscher dogs with familial dilated cardiomyopathy and sudden cardiac death. Human Genetics 138, 515-524 (2019).

68. L. Begay Rene, S. Graw, G. Sinagra, M. Merlo, D. Slavov, K. Gowan, L. Jones Kenneth, G. Barbati, A. Spezzacatene, F. Brun, A. Di Lenarda, E. Smith John, L. Granzier Henk, L. Mestroni, M. Taylor, n. null, Role of Titin Missense Variants in Dilated Cardiomyopathy. Journal of the American Heart Association 4, e002645 (2015).

69. Y. M. Pinto, P. M. Elliott, E. Arbustini, Y. Adler, A. Anastasakis, M. Böhm, D. Duboc, J. Gimeno, P. de Groote, M. Imazio, S. Heymans, K. Klingel, M. Komajda, G. Limongelli, A. Linhart, J. Mogensen, J. Moon, P. G. Pieper, P. M. Seferovic, S. Schueler, J. L. Zamorano, A. L. P. Caforio, P. Charron, Proposal for a revised definition of dilated cardiomyopathy, hypokinetic non-dilated cardiomyopathy, and its implications for clinical practice: a position statement of the ESC working group on myocardial and pericardial diseases. European Heart Journal 37, 1850-1858 (2016). 
1 70. L. Padrón-Barthe, M. Villalba-Orero, M. Gómez-Salinero Jesús, F. Domínguez, M. Román, J. Larrasa-Alonso, P. Ortiz-Sánchez, F. Martínez, M. López-Olañeta, E. Bonzón-Kulichenko, J. Vázquez, C. Martí-Gómez, J. Santiago Demetrio, B. Prados, G. Giovinazzo, V. Gómez-Gaviro María, S. Priori, P. Garcia-Pavia, E. Lara-Pezzi, Severe Cardiac Dysfunction and Death Caused by Arrhythmogenic Right Ventricular Cardiomyopathy Type 5 Are Improved by Inhibition of Glycogen Synthase Kinase-3ß. Circulation 140, 1188-1204 (2019).

71. J. Herron Todd, D. Rocha Andre Monteiro, F. Campbell Katherine, D. Ponce-Balbuena, B. C. Willis, G. Guerrero-Serna, Q. Liu, M. Klos, H. Musa, M. Zarzoso, A. Bizy, J. Furness, J. Anumonwo, S. Mironov, J. Jalife, Extracellular Matrix-Mediated Maturation of Human Pluripotent Stem Cell-Derived Cardiac Monolayer Structure and Electrophysiological Function. Circulation: Arrhythmia and Electrophysiology 9, e003638 (2016).

72. L. Sala, J. van Meer Berend, G. J. Tertoolen Leon, J. Bakkers, M. Bellin, P. Davis Richard, C. Denning, A. E. Dieben Michel, T. Eschenhagen, E. Giacomelli, C. Grandela, A. Hansen, R. Holman Eduard, R. M. Jongbloed Monique, M. Kamel Sarah, D. Koopman Charlotte, Q. Lachaud, I. Mannhardt, P. H. Mol Mervyn, D. Mosqueira, V. Orlova Valeria, R. Passier, C. Ribeiro Marcelo, U. Saleem, L. Smith Godfrey, L. Burton Francis, L. Mummery Christine, MUSCLEMOTION. Circulation Research 122, e5-e16 (2018).

73. C. Pimenta-Lopes, C. Suay-Corredera, D. Velázquez-Carreras, D. Sánchez-Ortiz, J. AlegreCebollada, Concurrent atomic force spectroscopy. Communications Physics 2, 91 (2019).

74. E. Gasteiger, C. Hoogland, A. Gattiker, S. e. Duvaud, M. R. Wilkins, R. D. Appel, A. Bairoch, in The Proteomics Protocols Handbook, J. M. Walker, Ed. (Humana Press, Totowa, NJ, 2005), pp. 571-607. 
Acknowledgements:

3 We thank CNIC's Pluripotent Cell Technology Unit for excellent technical support. Funding: This work was supported by the Ministerio de Ciencia e Innovación grants BIO2014-54768-P, BIO201783640-P, and RYC-2014-16604 to JAC, and RTI2018-096961-B-I00 to ELP, the Regional Government of Madrid grants S2018/NMT-4443 and PEJ16/MED/TL-1593 to JAC, the Instituto de Salud Carlos III (ISCIII) grants PI17/01941 to PGP and CPII14/00027 to ELP, and by a Mutual Medical Research Award 2017 to FD. We acknowledge funding from the European Research Area Network on Cardiovascular Disease through grants Genprovic to PGP (ISCIII- AC16/0014) and MINOTAUR to SS (The Austrian Science Fund - FWF, I3301-B31) and JAC (ISCIII-AC16/00045). We acknowledge support from the RD012/0042/0066 ISCIII grant to PGP and ELP. The CNIC is supported by ISCIII, the Ministerio de Ciencia e Innovación and the Pro CNIC Foundation, and is a Severo Ochoa Center of Excellence (SEV-2015-0505). IMM was the recipient of a CNIC-ACCIONA Masters Fellowship. CSC is the recipient of an FPI-SO predoctoral fellowship BES-2016-076638. Author contributions: PGP and JAC conceived and led the project. EHG, IMM, CSC, MRP, DG and RPJ analyzed titin sequence. EHG, IMM, CSG, NV and CBC did in-gel determination of reversible cysteine oxidations. EHG, IMM, EBK, EC and JV did and analyzed mass spectrometry experiments. MA, SS, and PPR procured and characterized human heart samples. EHG, IMM, NV and DVC cloned, purified and analyzed recombinant proteins. EHG, AFT, JV and JAC did statistical analysis of experimental data. IMM did Monte Carlo simulations. FD, EGL, MCM, BB, AB, JPO, JMGA TBR,

21 TMH, IVO, JPF and PGP obtained and interpreted clinical and genetics data. LL, BP, FGA, GG, JAB and ELP obtained hiPSC data. EHG, FD, IMM, PGP and JAC drafted the manuscript with input from all authors. Competing interests: The authors declare no competing interests. Data and material 
1 List of Supplementary Materials:

2 Supplementary File S1,

3 Supplementary Figures S1-S5,

4 Supplementary Notes S1-S4,

5 Supplementary Tables S1-S5

6 References (75-77)

7 
A
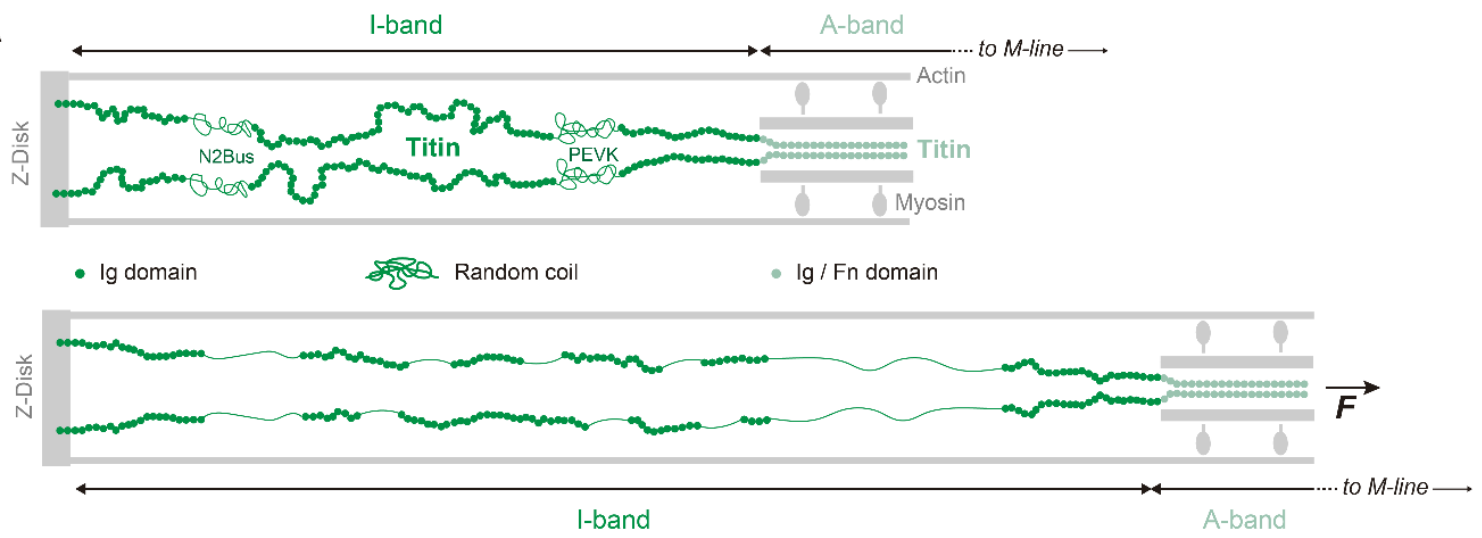

B
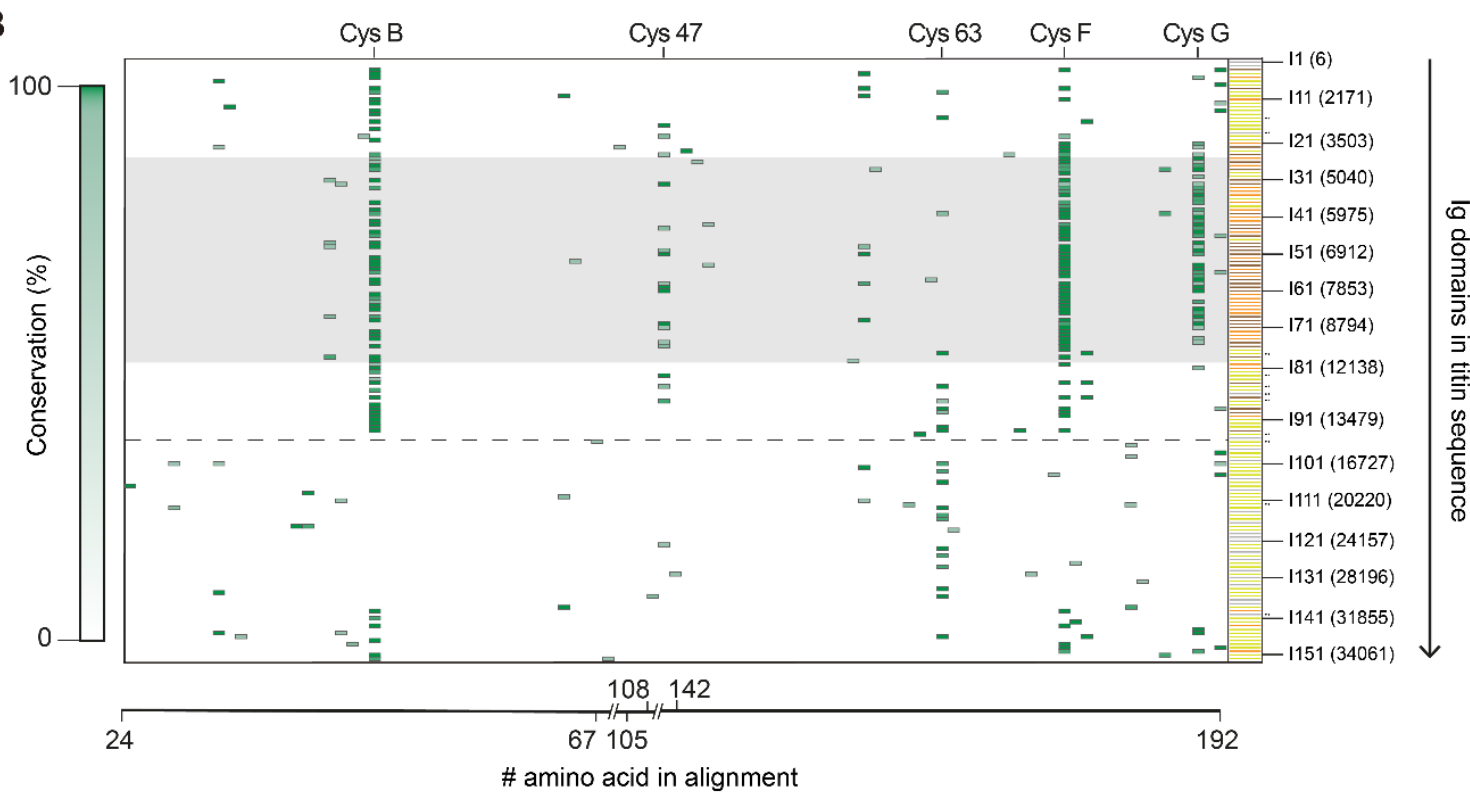

C

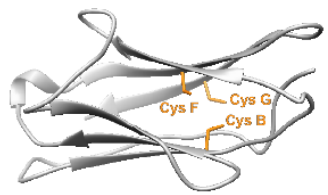

D

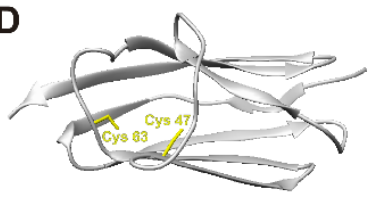

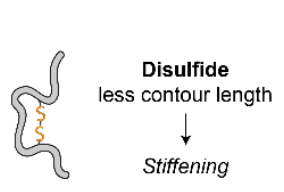

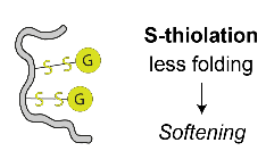

1

2 Figure 1. Evolutionary and structurally conserved cysteines in titin. A: Top: Representation of half

3 a sarcomere (not to scale), indicating the positions of the I-band (green) and the A-band (teal) of titin.

4 Immunoglobulin-like (Ig), random-coil (PEVK and N2Bus) and fibronectin III (Fn) domains are

5 shown. Bottom: Under mechanical force, the serially linked Ig domains and the random coil regions
E

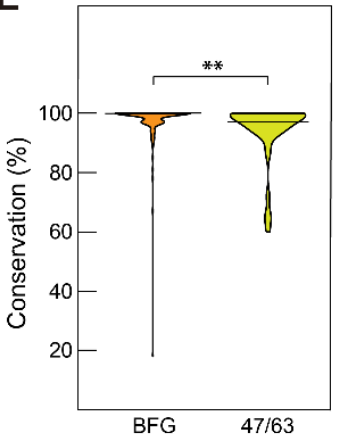

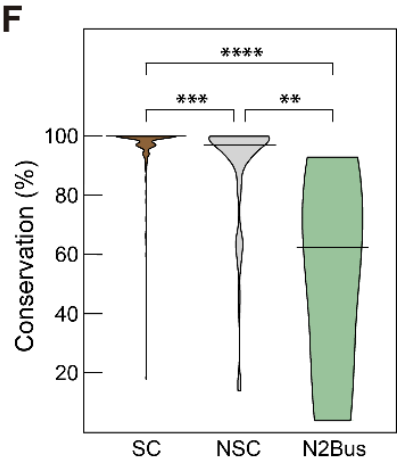


extend, and Ig domains unfold. B: Map of cysteine positions along the alignment of Ig domains of

2 human titin (breaks leave out Cys-free segments of the alignment). Structurally conserved positions B,

$347,63, \mathrm{~F}$ and $\mathrm{G}$ are indicated on top of the alignment. Positions are colored according to percentage of

4 evolutionary conservation, from 0 (white) to $100 \%$ (dark green). The number and positions of $\mathrm{Ig}$

5 domains according to Uniprot Q8WZ42-1 (N2BA isoform) are indicated on the right. Pairs of dots

6 mark Ig domains that are not annotated in Uniprot (21). Ig domains are represented in grey if they are

7 cysteine-free, in orange if they contain at least two of the triad, disulfide-competent cysteines B, F and

$8 \mathrm{G}(21)$, in yellow if they contain cysteines but no more than 1 cysteine B, F or G, and in brown if at

9 least two triad cysteines are present together with other cysteines. Spliced-out region in the N2B titin

10 isoform is indicated by the shaded grey area. The horizontal dashed line shows the boundary between

11 the I- and A-bands of titin (positions 14018-14019). C: 3D homology model of an Ig domain containing

12 the disulfide-competent CysB-CysF-CysG triad (I74, positions 9079-9168 from Uniprot entry

13 Q8WZ42). The reduced contour length of the disulfide-containing unfolded state leads to domain

14 stiffening. D: High-resolution structure of the traditionally named I27 domain of titin (also known as

15 I91, PDB code 1tit), which contains unpaired, S-thiolation-competent cysteines 47 and 63 (positions BFG $(n=153)$ and 47/63 ( $\mathrm{n}=40)$ cysteines in human titin. $\mathrm{p}(\mathrm{BFG}$ s $47 / 63)=0.0063$ (Mann-Whitney).

$21(\mathrm{n}=6)$ cysteines in human titin. $\mathrm{p}(\mathrm{SC}$ vs $\mathrm{NSC})=0.0001, \mathrm{p}(\mathrm{SC} v s \mathrm{~N} 2 \mathrm{Bus})<0.0001, \mathrm{p}(\mathrm{NSC} v s$

22 N2Bus)=0.0033 (Kruskal-Wallis and Dunn's multiple comparisons test). Evolutionary conservation

23 values in panels $\mathrm{E}$ and $\mathrm{F}$ were calculated from the alignment of 36 titin sequences from different species

24 (Supplementary Figure S1 and Supplementary File S1). Horizontal bars in violin plots indicate 25 median values. 
A
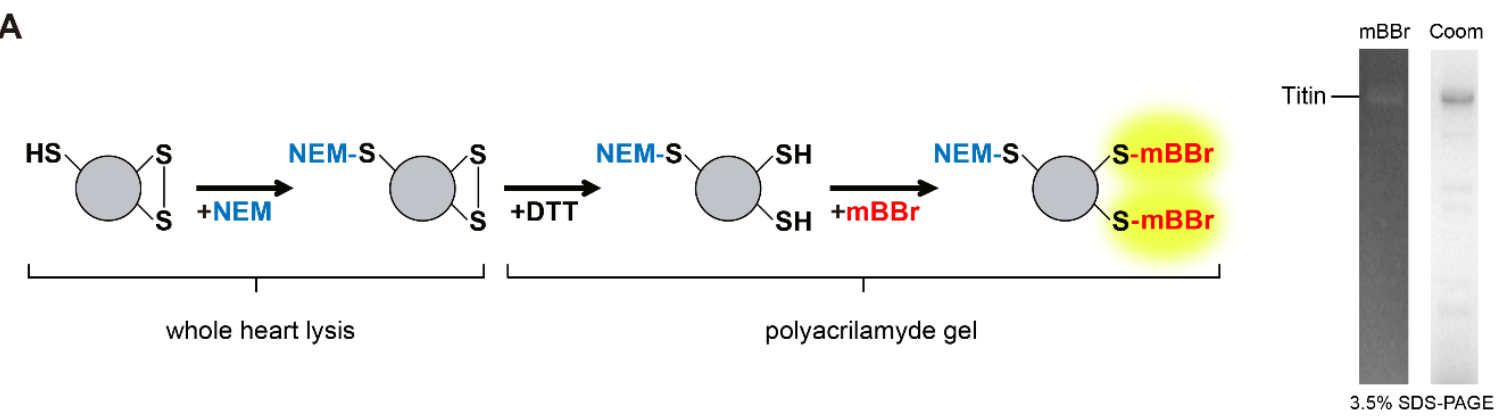

B

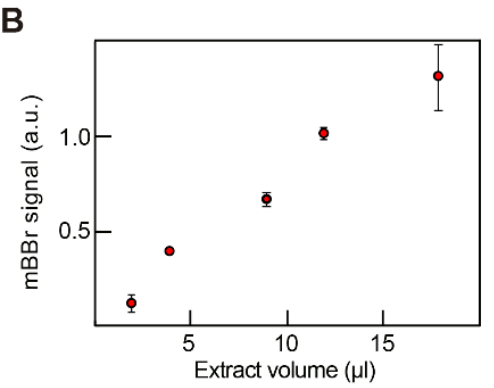

$\mathbf{E}$

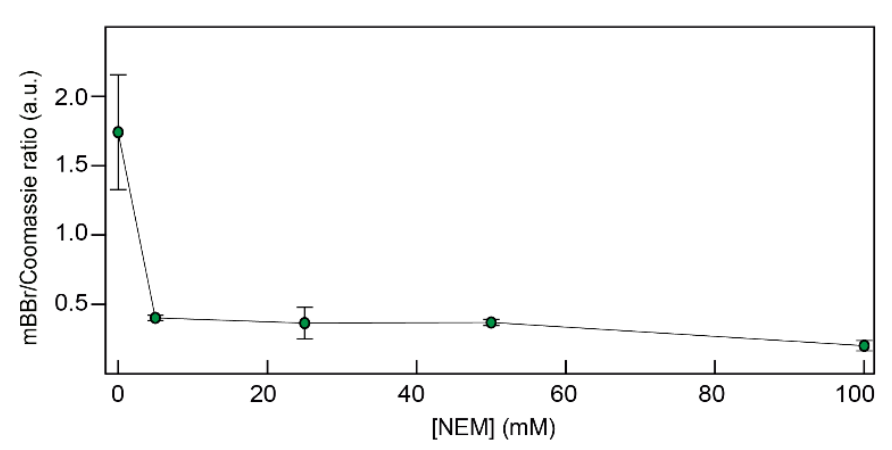

C

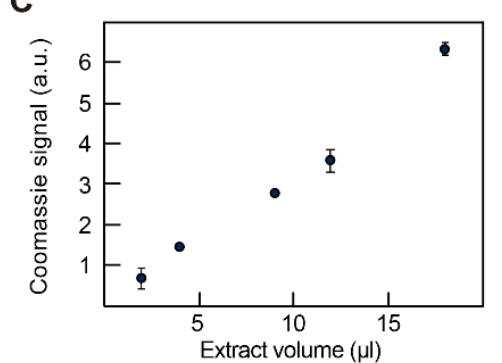

$\mathbf{F}$

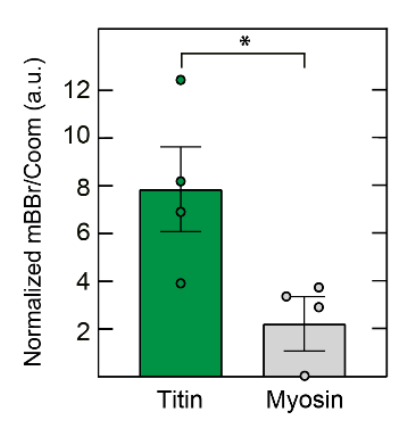

Figure 2. Titin is oxidized in basal conditions. A: Left: Reaction scheme to label reversibly oxidized thiols with $\mathrm{mBBr}$. Protein extracts in which reduced thiols have been initially blocked by NEM are run in an SDS-PAGE gel. Following electrophoresis, oxidized thiols are reduced by DTT and then labelled with the fluorescent probe $\mathrm{mBBr}$. Right: example SDS-PAGE gel used to quantify mBBr fluorescence from titin bands. Coomassie staining of the same gel is used for normalization. $\mathbf{B}, \mathbf{C}$ : The $\mathrm{mBBr}$ and Coomassie signals originating from titin are linearly dependent on the amount of lysate loaded in SDSPAGE gels (all data points are the average of duplicates) (Supplementary Figure S2). D: Final 11 the concentration of NEM needed to completely block initially reduced titin thiols. Duplicates were 
1 performed for each NEM concentration. F: Quantification of the oxidation of titin and myosin in four

2 different mouse hearts using 3.5\% and 12\% SDS-PAGE gels, respectively. The volume of lysate

3 analyzed was adjusted so that both titin $(15 \mu \mathrm{l})$ and myosin $(3 \mu \mathrm{l})$ were in the linear range of detection

4 for Coomassie. Oxidation signal is normalized by the density of cysteines of each protein (13.3

5 Cys/1000 amino acid for titin, 7.2 Cys/1000 amino acid for myosin, see Methods). $\mathrm{n}=4$ animals, $6 \mathrm{p}=0.0286$ (Mann-Whitney). 


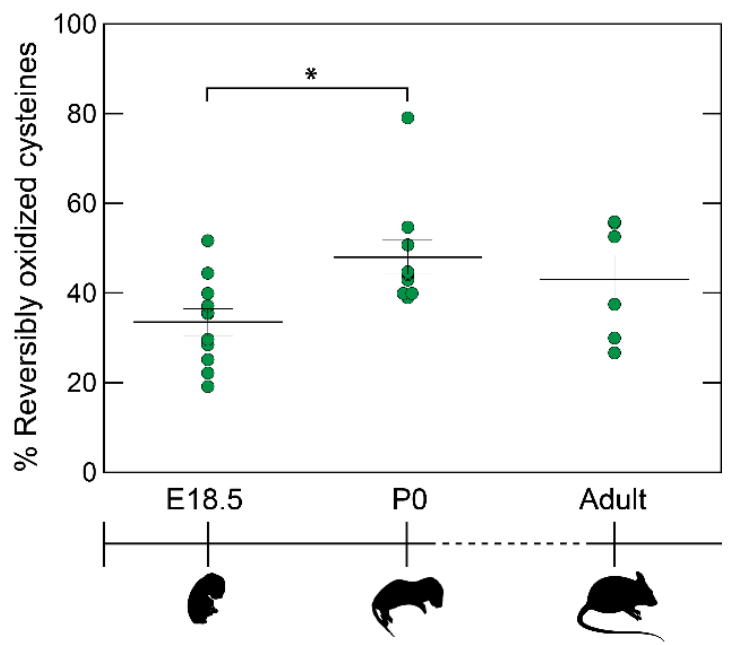

2 Figure 3. Titin oxidation is increased upon birth. Reversible oxidation of titin cysteines in samples

3 from 18.5-days embryos $(\mathrm{E} 18.5, \mathrm{n}=11)$, newborn $(\mathrm{P} 0, \mathrm{n}=10)$ and adult mice $(\mathrm{n}=6)$. Density of

4 reversibly oxidized cysteines (Supplementary Figure S3A) is compared to the total density of

5 cysteines according to the percentages of each titin isoform (14.6 Cys/1000 amino acid for N2BA and

$6 \quad 13.3$ Cys/1000 amino acid for N2B, Supplementary Figure S3Bn; see Methods). p(E18.5 vs P0) =

$7 \quad 0.0259, \mathrm{p}(\mathrm{E} 18.5 v s$ Adult $)=0.259, \mathrm{p}(\mathrm{P} 0$ vs Adult $)>0.999$ (Kruskal-Wallis and Dunn's multiple

8 comparisons test). 
A



C

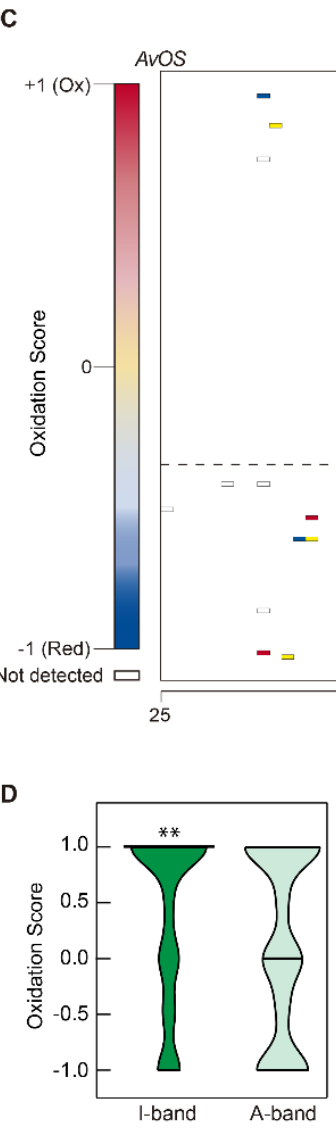

B



$\begin{array}{lllll}\text { Cys B } & \text { Cys } 47 & \text { Cys } 63 & \text { Cys } F & \text { Cys } G\end{array}$

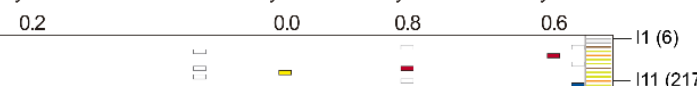

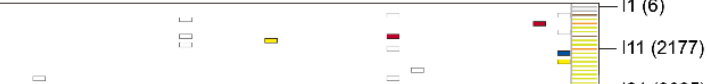

$-=\quad \bar{E}^{-}$

-
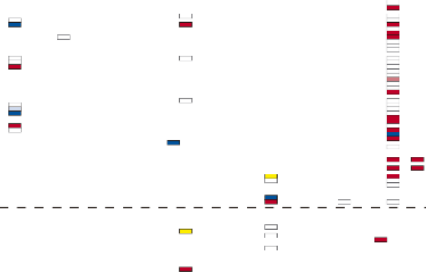

- -

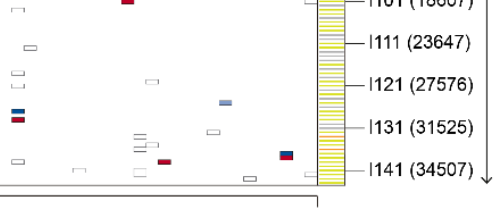

amino acid in alignment

E

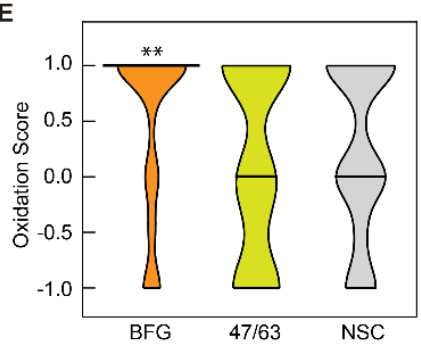

F

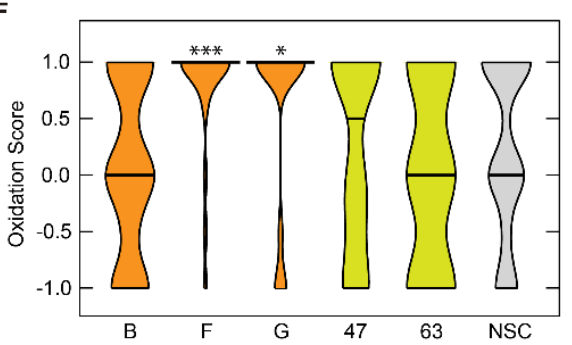

2 Figure 4. Landscape of cysteine oxidation in murine postnatal (P0) cardiac titin. A: Fraction of

3

$4 \quad$ N2BA titin isoform) when the MS/MS spectra are searched against a mouse proteome database

containing common contaminants (cRAP). B: Aggregated itin coverage obtained after searching against the mouse proteome ( $\mathrm{n}=3$ samples; yellow and green, peptides detected with FDR $<5 \%$ and $<1 \%$, respectively). C: Oxidation Score (OS) map for every cysteine position in the alignment of Ig domains of mouse titin, according to the color code indicated on the left (red, oxidized; blue, reduced). Average OS (AvOS) for cysteines 47, 63 and the BFG triad across all Ig domains are indicated. Cysteines shown in white were not identified and do not contribute to calculation of OS. The horizontal dashed line shows the boundary between the I- and A-bands of titin (positions 14880-14881 in Uniprot 
1 A2ASS6-1). The number and positions of Ig domains are indicated on the right. Ig domains are

2 classified and colored as in Figure 1. D: Violin-plot distributions of OS for cysteines belonging to the

3 I- $(\mathrm{n}=97)$ and A- $(\mathrm{n}=123)$ bands of murine titin. $\mathrm{p}(\mathrm{OS}>0, \mathrm{I}-\mathrm{band})=0.0054, \mathrm{p}(\mathrm{OS}>0, \mathrm{~A}-\mathrm{band})=0.9886$

4 (hypergeometric test). E: Violin-plot distributions of OS for cysteines BFG (n=61), 47/63 (n=14) and

5 non-structurally conserved $(\mathrm{NSC}, \mathrm{n}=33) . \mathrm{p}(\mathrm{OS}>0, \mathrm{BFG})=0.0054, \mathrm{p}(\mathrm{OS}>0,47 / 63)=0.8137, \mathrm{p}(\mathrm{OS}>0$,

6 NSC) $=0.9268$ (hypergeometric test). F: Violin-plot distributions of OS for cysteines B $(\mathrm{n}=21), \mathrm{F}$

$7(\mathrm{n}=23), \mathrm{G}(\mathrm{n}=17), 47(\mathrm{n}=8), 63(\mathrm{n}=6)$ and NSC $(\mathrm{n}=33) . \mathrm{p}(\mathrm{OS}>0, \mathrm{~B})=0.9593, \mathrm{p}(\mathrm{OS}>0, \mathrm{~F})=0.0001$,

$8 \mathrm{p}(\mathrm{OS}>0, \mathrm{G})=0.0200, \mathrm{p}(\mathrm{OS}>0,47)=0.53365, \mathrm{p}(\mathrm{OS}>0,63)=0.7894, \mathrm{p}(\mathrm{OS}>0, \mathrm{NSC})=0.9268$

9 (hypergeometric test). Horizontal bars in violin plots indicate the median values. 
A

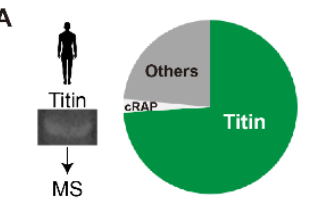

B

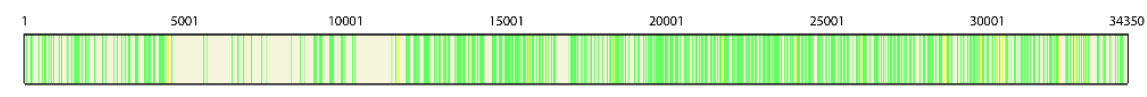

C

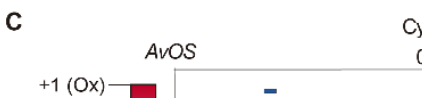

Cys B



0.0

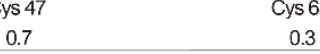

Cys $63 \quad$ Cys $\mathrm{G}$



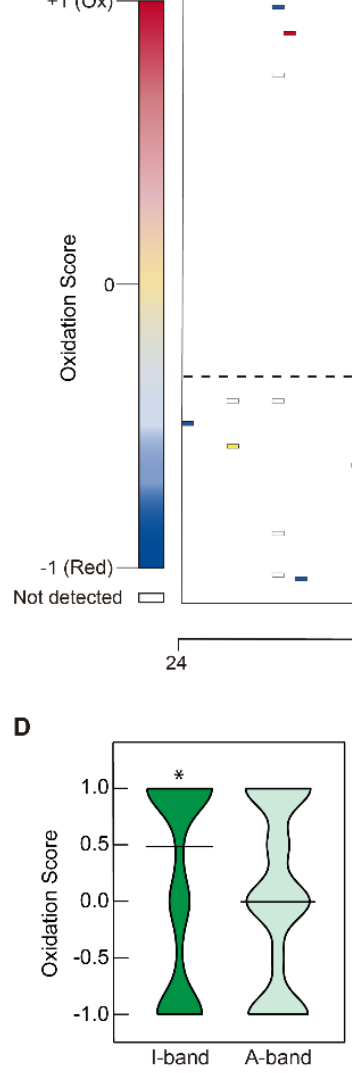
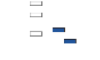

$=$ E

$=$ 長

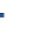

2 Figure 5. Landscape of cysteine oxidation in adult human cardiac titin. A: Fraction of identified

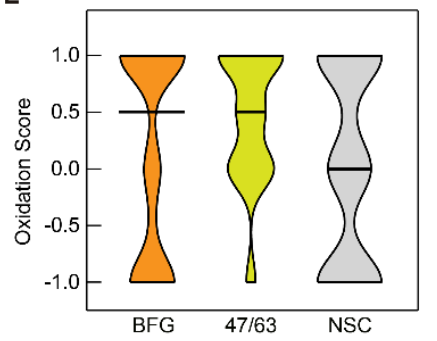

$\mathbf{F}$

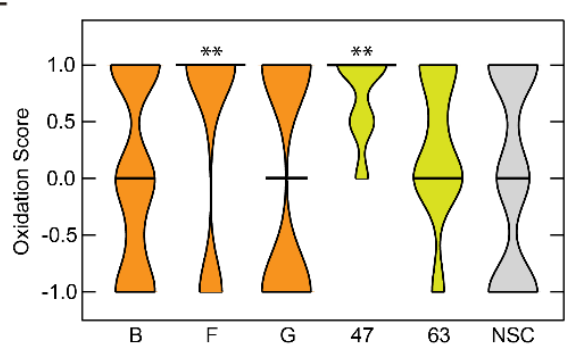

3 peptides belonging to titin (reference sequence Uniprot Q8WZ42-1, which corresponds to the long

4 N2BA titin isoform) when the MS/MS spectra are searched against a database containing the human

5 proteome and common contaminants (cRAP). B: Aggregated titin coverage obtained after searching

6 against the human proteome $(n=2$ samples; yellow and green, peptides detected with FDR $<5 \%$ and $<1 \%$, respectively). C: Oxidation Score (OS) map for every cysteine position in the alignment of Ig domains of human titin, according to the color code indicated on the left (red, oxidized; blue, reduced). Average OS $(A v O S)$ for cysteines 47, 63 and the B/F/G triad across all Ig domains are indicated. Cysteines shown in white were not identified and do not contribute to calculation of OS. Red arrow points to Cys3575, a target of DCM-linked mutations. The dashed line shows the boundary between 
1 the I- and A-bands of titin (positions 14018-14019 in Uniprot Q8WZ42-1). The number and positions

2 of Ig domains are indicated on the right. Pairs of dots mark Ig domains that are not annotated in Uniprot.

3 Ig domains are classified and colored as in Figure 1. D: Violin-plot distributions of OS for cysteines

4 belonging to the I- $(n=77)$ and A- $(n=122)$ bands of human titin. $\mathrm{p}(\mathrm{OS}>0$, I-band $)=0.0435, \mathrm{p}(\mathrm{OS}>0$,

5 A-band) $=0.9220$ (hypergeometric test). E: Violin-plot distributions of oxidation scores for cysteines

6 BFG ( $n=57), 47 / 63(n=16)$ and non-structurally conserved (NSC, $n=33) . p(O S>0, B F G)=0.2746$,

$7 \mathrm{p}(\mathrm{OS}>0,47 / 63)=0.0745, \mathrm{p}(\mathrm{OS}>0, \mathrm{NSC})=0.8705$ (hypergeometric test). F: Violin-plot distributions

8 of oxidation scores for cysteines B $(n=25), F(n=20), G(n=12), 47(n=7), 63(n=9)$ and NSC ( $n=33)$ in

9 human cardiac titin. $\mathrm{p}(\mathrm{OS}>0, \mathrm{~B})=0.8974, \mathrm{p}(\mathrm{OS}>0, \mathrm{~F})=0.0093, \mathrm{p}(\mathrm{OS}>0, \mathrm{G})=0.3537, \mathrm{p}(\mathrm{OS}>0,47)=$

$100.0055, \mathrm{p}(\mathrm{OS}>0,63)=0.4759, \mathrm{p}(\mathrm{OS}>0, \mathrm{NSC})=0.8705$ (hypergeometric test). Horizontal bars in violin

11 plots indicate the median values.

12 
A

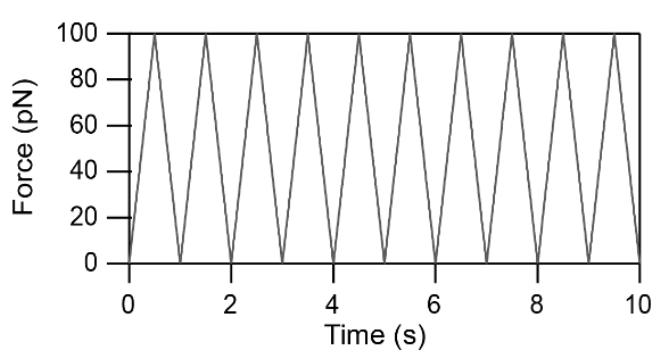

C

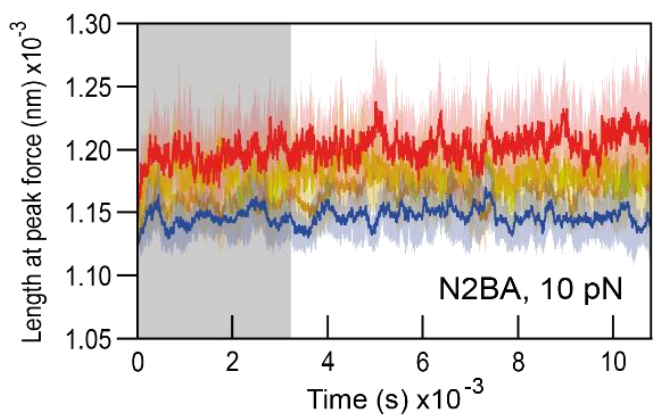

E

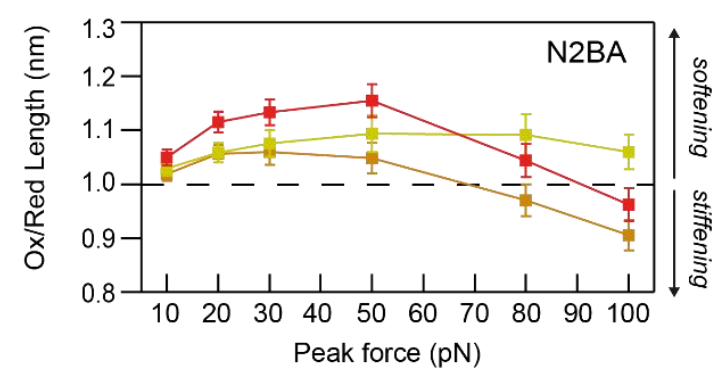

$\mathbf{G}$

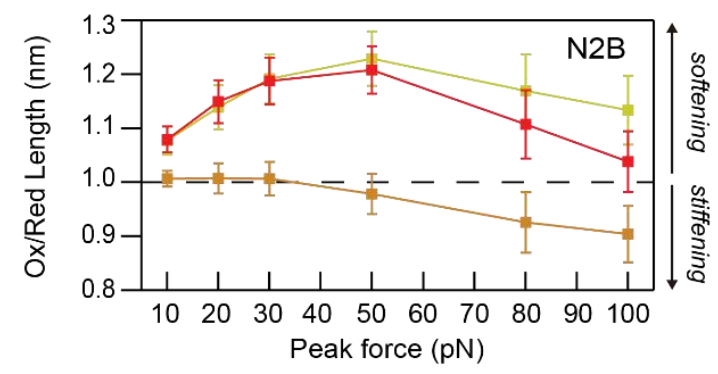

1
B

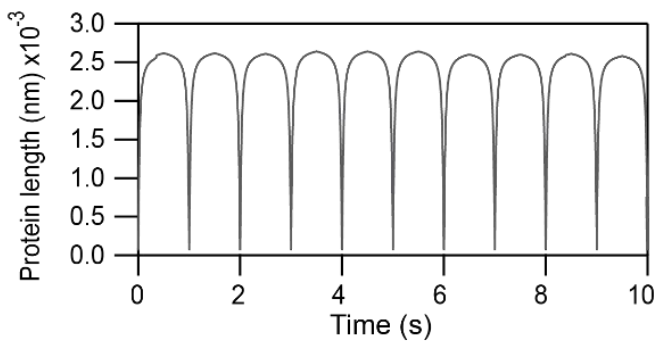

D

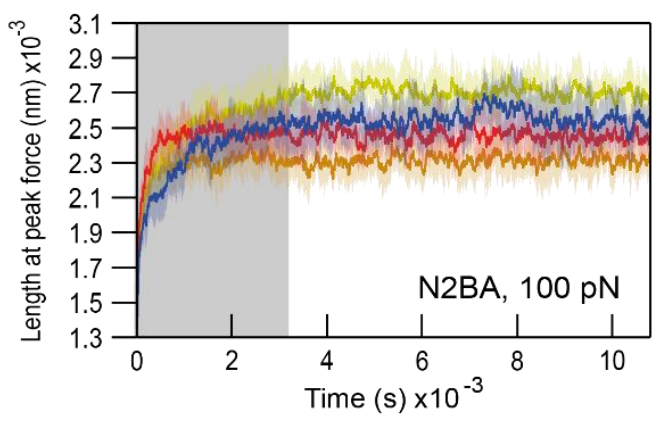

$\mathbf{F}$

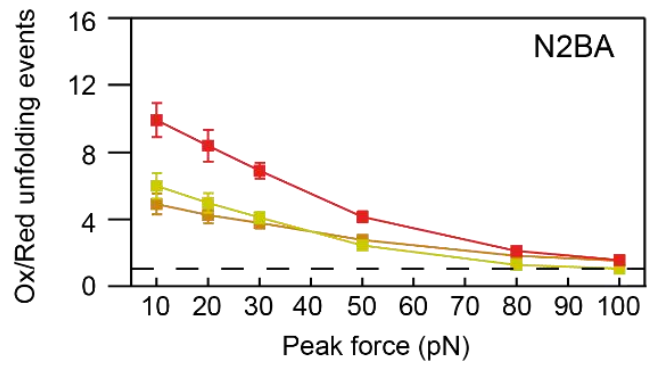

H



Figure 6. Effects of reversible cysteine oxidations on titin mechanics. A: Monte Carlo simulations

subject virtual titins to a $1 \mathrm{~Hz}$ oscillating triangular force pulse to a predefined peak force $(100 \mathrm{pN}$ in the example shown). B: Example of the length of reduced N2BA titin when pulled to a $100 \mathrm{pN}$ peak force (10 seconds of simulation after reaching steady state are shown). C,D: Length of N2BA titin at peak force during simulations in which titin is reduced, or oxidized by disulfides, S-thiolation adducts or both (color code is indicated at the bottom; peak forces are indicated in the insets). Graphs show the 
1 average of 10 independent simulations and SD is indicated by shaded areas. For subsequent analyses,

2 we considered times longer than 1 hour, in which the length of titin fluctuates around steady state values

3 (region outside the grey shading). E: Ratio of oxidized $v s$ reduced N2BA titin length at different peak

4 forces. F: Ratio of oxidized $v s$ reduced N2BA unfolding events in simulations at different peak forces.

5 G: Ratio of oxidized $v s$ reduced N2B titin length at different peak forces. H: Ratio of oxidized $v s$ 6 reduced N2B unfolding events in simulations at different peak forces. In panels E-H, n= 10 simulations; 7 error bars: SD. 
A


B

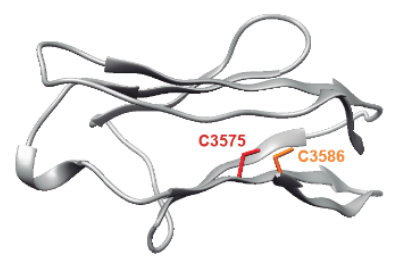

C



$\checkmark$ Proband

- Confirmed non carriers

+ Confirmed carriers

- Affected (DCM)

$\mathbf{F}$

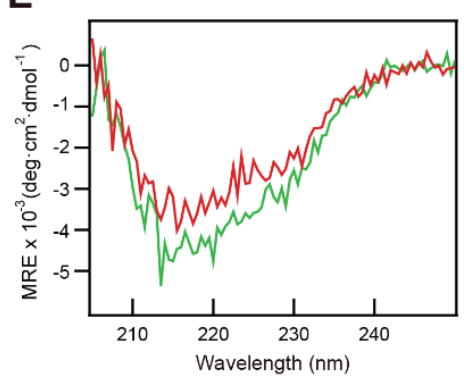

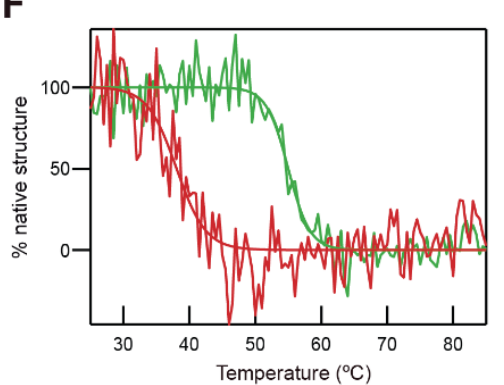

Figure 7. Mutations in conserved Cys3575 cause dilated cardiomyopathy. A: Pedigree of a large

Spanish family with 14 TTN p.Cys3575Ser carriers (12 with DCM, penetrance 86\%) and 22 noncarriers. B: Homology model of the I21 domain of titin, highlighting its two cysteine residues. C: Pedigree of a Danish family with the TTN p.Cys3575Arg variant, with 3 carriers ( 2 with DCM, penetrance 67\%) and 1 non-carrier. D: Box-and-whiskers plots showing amplitude of contraction of WT (green, $\mathrm{n}=87$ ) and homozygous Cys3575Ser (red, $\mathrm{n}=119$ ) hiPSC-induced cardiomyocytes ( $\mathrm{p}=0.0001$, Mann-Whitney). Box encompasses data between quartiles 1 and 3 and horizontal lines represent the median of the distributions. Whiskers and outliers were calculated using Tukey's method as implemented in Graph Pad. E: Far-UV circular dichroism spectra at $25^{\circ} \mathrm{C}$ of WT (green) and 
bioRxiv preprint doi: https://doi.org/10.1101/2020.09.05.282913; this version posted September 6, 2020. The copyright holder for this preprint (which was not certified by peer review) is the author/funder. All rights reserved. No reuse allowed without permission.

1 Cys3575Ser (red) recombinant I21 protein domains. F: Thermal unfolding curves of recombinant I21

2 WT (green) and Cys3575Ser (red) domains. Sigmoidal fits to the data are shown. 